\title{
Through-the-Thickness Stress Distributions near Edges of Composite Laminates using Stress Recovery Scheme and Third Order Shear and Normal Deformable Theory
}

\author{
P. H. Shah and R. C. Batra* \\ Department of Biomedical Engineering and Mechanics, M/C 0219, \\ Virginia Polytechnic Institute and State University, Blacksburg, VA 24061, USA \\ *Corresponding author; Tel. +1-540-231-6051; Fax:+1-540-231-4564; Email: rbatra@vt.edu
}

\begin{abstract}
The reliable prediction of failure in laminated plates using stress based failure theories requires accurate evaluation of stresses. Noting that stresses are likely to be singular near laminate edges, we explore here whether or not a third order shear and normal deformable plate theory (TSNDT) and the commonly used stress recovery scheme (SRS) enables one to accurately compute stresses near edges of a composite laminate deformed statically with surface tractions applied on its major surfaces. In the TSNDT the three displacement components at a point are expressed as complete polynomials of degree three in the thickness coordinate. For laminated plates, we use a single layer TSNDT and the SRS to compute through-the-thickness distribution of transverse normal and transverse shear stresses. However, for monolithic plates stresses are obtained directly from the constitutive relations. Using in-house developed software based on the finite element (FE) formulation of the problem, we study seven example problems for plates subjected to different boundary conditions at the edges and various loads such as combined normal and tangential tractions uniformly distributed on the major surfaces, non-uniform pressure acting on a part of the major surface, and sinusoidal normal tractions. For each problem studied stresses computed using the TSNDT are compared with those obtained by analyzing 3-dimensional (3-D) linear elastic deformations with either the FE commercial software, ABAQUS, or the analytical solution reported in the literature. The significance of the present work is in investigating whether or not the TSNDT and the SRS give accurate values of stresses near edges of moderately thick laminates. It is found that for some thick laminates (span/thickness $=5$ ), stress distributions near an edge predicted by the TSNDT coupled with the SRS differ by $40 \%$ from those computed by analyzing the 3-D deformations.
\end{abstract}

Keywords: TSNDT, laminated plates, static deformations, stress recovery scheme 


\section{Introduction}

A great deal of research has been devoted to developing theories for analyzing deformations of plates. The assumptions made in the classical plate theory (CPT) or the Kirchhoff [1] thin plate theory are: (i) a straight line perpendicular to the mid-surface (transverse normal) before deformation remains straight and normal to the mid-surface after deformation, and (ii) the plate thickness remains unchanged. These assumptions imply that the transverse normal and shear strains are zero. The condition of null transverse normal strain is usually identically satisfied by modifying the stress-strain relation. This theory may not give accurate distributions of transverse normal and shear stresses and strains for moderately thick plates (thickness/span > 1/10). This shortcoming is partially overcome by using a stress recovery scheme (SRS) in which equilibrium equations are integrated along the thickness direction to compute transverse normal and shear stresses by starting from the surface tractions applied on one of the major surfaces of the plate. Many works have successfully applied the SRS to determine stresses at interior points of a plate.

Rather than making kinematic assumptions (i.e., assuming a displacement field) for a plate E. Cosserat and F. Cosserat [2] regarded a plate as a surface with a director (3dimensional vector) attached to each point of the plate. Deformations of the director account for transverse shear and transverse normal deformations of the plate. Constraining the director to be always a unit vector that stays normal to the mid-surface of the plate is equivalent to using the Kirchhoff plate theory. This theory is usually called the direct plate theory since no assumptions on through-the-thickness variations of displacements are made. Ericksen [3] has studied plane waves in plates by using such a theory. Koiter and Simmonds [4], Naghdi [5], Antman [6], Leissa [7] and Carrerra [8] amongst others have reviewed the literature on plate theories. There are numerous other review articles and books on plate theories that are not mentioned here for the sake of brevity.

Mindlin and Medick [9] expressed the three displacement components at a point as an infinite series in terms of two functions one of which is the Legendre polynomials of the thickness coordinate, $\mathrm{z}$, and the other a function of the in-plane coordinates, (x,y), of a point on the plate mid-surface. They used the principle of virtual work to derive equations for the plate theory of different order. The plate theory in which terms up to the order $\mathrm{z}^{\mathrm{K}}$ are kept is usually called the $\mathrm{K}^{\text {th }}$ order theory. They used the $2^{\text {nd }}$ order theory to study vibrations of a 
plate. It is hard to characterize the order of the plate theory when trigonometric functions in the $\mathrm{z}$-coordinate rather than the Legendre polynomials are used to expand displacements.

Teresi and Tiero [10] deduced plate theories by seeking stationary points in suitable subspaces of the functional spaces in which the potential energy, the complementary energy and the Hellinger-Prange-Reissner (HPR) functionals are defined. For an isotropic plate they showed that these methods give different values of the flexural and shear rigidities. Advantages of using the HPR principle include making independent assumptions on the stress and displacement fields, and exactly satisfying traction boundary conditions prescribed on the major surfaces of the plate. Vidoli and Batra [11] and Batra and Vidoli [12] used the HPR principle to deduce plate theories of different orders for piezoelectric and orthotropic linear elastic plates. When constitutive relations for the plate theory are derived by using the HPR principle, they called the plate theory "mixed". However, when these equations are deduced from the assumed displacement field and the constitutive relations of the 3dimensional (3-D) linear elasticity theory, they called it "compatible". We note that most plate theories studied in the literature are compatible. Batra and Vidoli [12] showed that for infinitesimal deformations of a very thick cantilever beam (length/thickness $=2)$ the mixed plate theory gives stress distributions in close agreement with the analytical solution of the problem than that from the compatible plate theory of the same order. Batra et al. [13] used the plate theories of different orders up to $K=5$ to study wave propagation in thick plates.

As has been pointed by Carrera [14] and also evidenced by numerical solutions provided by Qian et al. $[15,16]$ the order of the plate theory that gives stresses and displacements close to those predicted by the 3-D linear elasticity theory (LET) depends upon the problem being studied, i.e., the plate geometry, applied loads, and initial and boundary conditions. Said differently, there is no universal plate theory that gives accurate results for all plate problems. Truncating the series expansion for the displacements and stresses introduces errors that are not easy to quantify a priori.

Due to their high specific properties, laminated composite structures are widely used in construction, automobile, aerospace and marine applications. In general, the theories developed for analyzing deformations of laminated composite structures can be divided into two major categories, namely, equivalent single layer (ESL) theories and layerwise zig-zag theories. In the ESL the displacement field assumed is the same as in a monolithic plate, thus 
the number of unknowns is independent of the number of layers in the laminate. The challenge is to satisfy the continuity of surface tractions at interfaces between adjoining layers. The simplest ESL theory is the classical laminated plate theory (CLPT) [17] which is an extension of the CPT to thin laminated plates. For thick laminated plates, higher order ESL theories [18-20] can provide more accurate response than that predicted by the CLPT. However, the transverse normal and shear stresses computed using ESL theories may not be accurate and continuous across the interface between the adjoining layers. This is remedied using a SRS. The layerwise zig-zag theories [21-26] satisfy the continuity of surface tractions at the layer interfaces but introduce considerably more unknowns into the problem.

Carrera in a review paper [27] has pointed out that the first zig-zag theory for multilayered structures was proposed by Lekhnitskii [22] who studied deformations of laminated composite beams. Ren [28, 29] extended Lekhnitskii's theory to study deformations of anisotropic plates. Ren and Owen [30] applied this theory to study vibrations and buckling of laminated plates. Following the Reissner-Mindlin plate theory, Ambartsumian [31-32] proposed a zig-zag theory to analyze deformations of layered anisotropic plates and shells. Whitney [33] extended Ambartsumian's theory to general anisotropic symmetric and nonsymmetric plates, and Rath and Das [34] further extended Whitney's work to doubly curved shells for dynamic problems. For more details and historic evaluation of various ESL and zig-zag theories, readers should study review papers by Carrera [27], Ambartsumian [35, 36], Ghugal and Shimpi [37], Reddy [38], and Reddy and Arciniega [39].

The SRSs employed by various investigators can be classified, in general, as one-step and two-step methods [40]. In the one-step method, the in-plane stresses are obtained using constitutive relations and the plate theory displacements, and the transverse stresses are computed by integrating the equilibrium equations over the laminate thickness. For example, Pagano [41] obtained closed-form solutions for inter-laminar stresses in a simply supported laminated plate by integrating equilibrium equations of the 3-D elasticity over the plate thickness. He first obtained analytical expressions for intra-laminar stresses based on the CLPT and differentiated them with respect to spatial coordinates to compute derivatives of stresses for use in the equilibrium equations. The inter-laminar stresses thus computed agreed well with the analytical solution of the problem based on the 3-D linear elasticity theory (3-D LET). Rohwer [42] also showed that solving the equations of equilibrium locally yields acceptable values of transverse shear stresses with the CLPT or a higher order plate theory. 
The two-step methods use iterative techniques to compute inter-laminar transverse stresses. For example, Noor et al. [43, 44] used a predictor-corrector approach with the first order shear deformable theory (FSDT) [8] to compute transverse stresses from the equilibrium equations. In the predictor phase, the in-plane stresses are obtained using the FSDT with a shear corrector factor, and the transverse stresses are determined from the equilibrium equations and the corresponding strain energies are computed. The corrector phase uses predicted values of either the strain energy due to shear deformations or shear stresses to refine either the shear correction factor or through-the-thickness distributions of displacements, respectively, until the convergence is obtained. Malik and Noor [45] employed a slightly different predictor-corrector approach in which displacements and strains are refined until the convergence is obtained and then using the constitutive relations in-plane stresses are obtained, and from the equilibrium equations transverse stresses are computed. Chaudhuri and Seide [46] rather than using the 3-D equations of equilibrium used 1-D quadratic shape functions through the thickness of each layer to compute transverse shear stress distributions in the laminate. Engelstad et al. [47], Byun and Kapania [48] and Hartman et al. [49] extended the use of one-step SRSs to geometrically non-linear problems while Park et al. [50] used a two-step SRS similar to that employed by Noor et al. [43] to predict interlaminar stresses in the laminated panels undergoing finite rotations.

Here we study static infinitesimal deformations of laminated composite plates using the compatible third-order shear and normal deformable theory (TSNDT). The assumed displacement field is continuous through the laminate thickness. For a monolithic plate, we find stresses directly from the constitutive relations. However, for multi-layered plates, we obtain in-plane stresses from the constitutive relations and compute transverse stresses by using the one-step SRS. No shear correction factor is used. We numerically solve equations of the plate theory by the FEM with our in-house code, and compare computed results with the corresponding analytical solutions or finite element (FE) results based on the 3-D LET obtained with the commercial software ABAQUS. It is found that for some laminates the SRS coupled with the TSNDT displacement field gives stresses at points near the laminate edges that differ by as much as $40 \%$ from their analytical values. However, stresses at interior points are quite accurate and differ by at most $7 \%$ from their analytical values. Thus studying failure and damage initiation and propagation in laminated composites using an ESL may not be desirable even though it is computationally less expensive. 


\section{Formulation of the Problem}

Consider a laminated plate, shown in Fig. 1, composed of $\mathrm{N}$ layers of not necessarily the same thickness. Each ply is made of a homogeneous, orthotropic and linear elastic material with adjacent layers assumed to be perfectly bonded to each other. Let $\left(\mathrm{X}_{1}, \mathrm{X}_{2}, \mathrm{X}_{3}\right)$ denote fixed rectangular Cartesian coordinate axes in the reference (undeformed) configuration of the laminated plate such that $\mathrm{X}_{3}=0$ represents the mid-surface, $\mathrm{h}$ the total thickness, and $a$ and $b$ lengths of sides along the $\mathrm{X}_{1}$ - and the $\mathrm{X}_{2}$ - directions, respectively. The vertical positions of the bottom and the top surfaces of layer $k$ are denoted by $\mathrm{h}_{k}$ and $\mathrm{h}_{k+1}$, respectively, with $k=1$ and $k=\mathrm{N}$ representing the bottom and the top layer of the laminate.

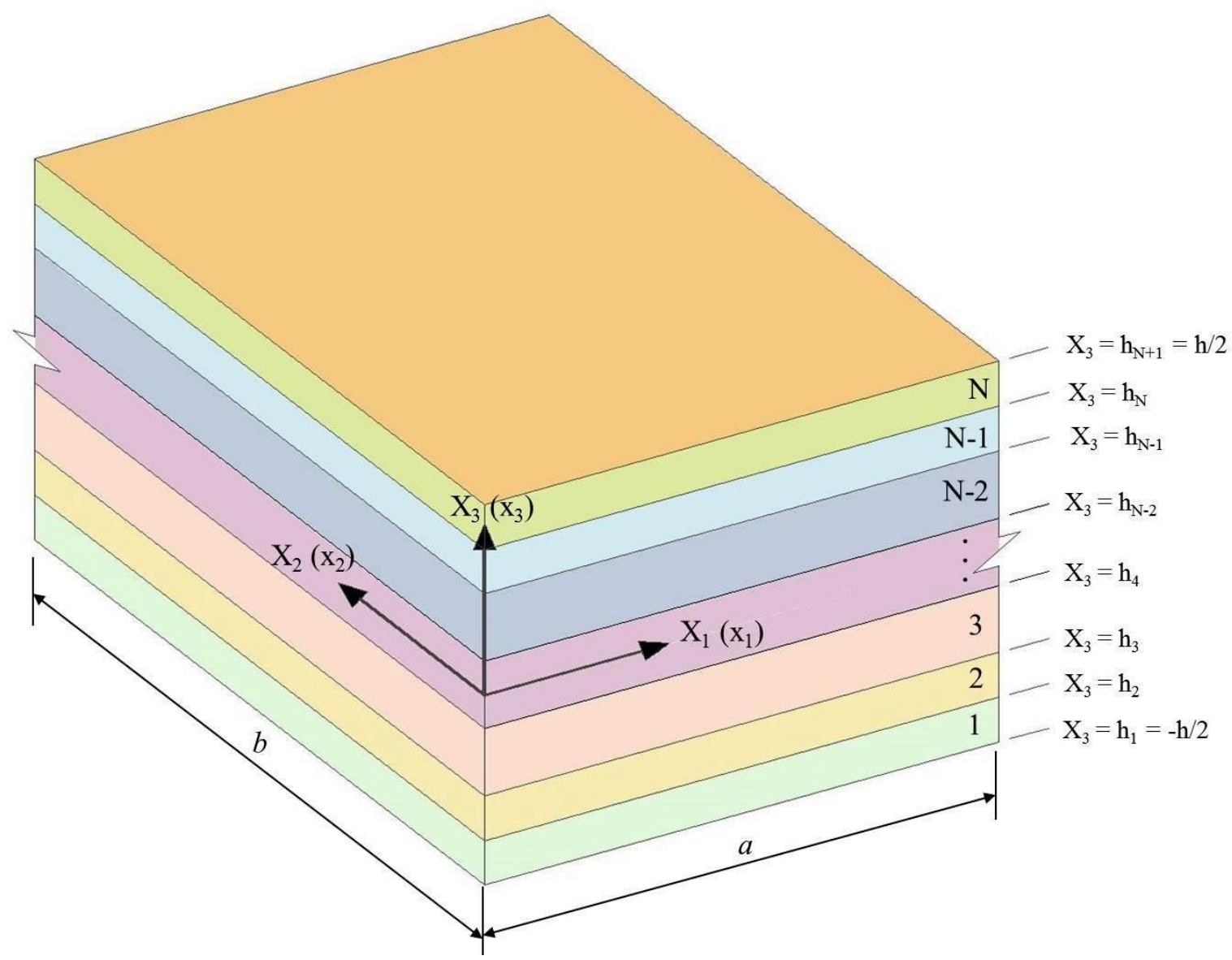

Fig. 1 Geometry and coordinate system for a laminated plate

Let $\mathbf{x}$ be the position vector of a point $\mathrm{p}$ in the current configuration that occupied place $\mathbf{X}$ in the reference configuration. The displacement $\mathbf{u}$ and components of the infinitesimal strain tensor $\epsilon_{\mathrm{ij}}$ at $\mathrm{p}$ are given, respectively, by $\mathbf{u}=\mathbf{x}-\mathbf{X}$ and 
$\epsilon_{\mathrm{ij}}=\frac{1}{2}\left[\frac{\partial \mathrm{u}_{\mathrm{i}}}{\partial \mathrm{X}_{\mathrm{j}}}+\frac{\partial \mathrm{u}_{\mathrm{j}}}{\partial \mathrm{X}_{\mathrm{i}}}\right](\mathrm{i}, \mathrm{j}=1,2,3)$

For infinitesimal deformations studied herein, displacements in Eq. (1) can also be differentiated with respect to $\mathbf{x}$ rather than $\mathbf{X}$. In the TSNDT the displacement, $u_{i}$, at a point is expressed as a complete polynomial of degree 3 in $\mathrm{X}_{3}$ that can be obtained by Taylor series expansion of $\mathrm{u}_{\mathrm{i}}$ in the thickness coordinate, $\mathrm{X}_{3}$, up to order 3 , i.e.,

$$
\begin{aligned}
& u_{i}\left(X_{1}, X_{2}, X_{3}\right)=u_{i j}\left(X_{1}, X_{2}\right) A_{j}\left(X_{3}\right) \quad(i=1,2,3 ; j=0,1,2,3), \\
& A_{j}=X_{3}^{j}, u_{i 0}=u_{i}\left(X_{1}, X_{2}, 0\right), u_{i 1}=\left.\frac{\partial u_{i}}{\partial X_{3}}\right|_{X_{3}=0}, 2 u_{i 2}=\left.\frac{\partial^{2} u_{i}}{\partial X_{3}^{2}}\right|_{X_{3}=0}, 6 u_{i 3}=\left.\frac{\partial^{3} u_{i}}{\partial X_{3}^{3}}\right|_{X_{3}=0} .
\end{aligned}
$$

Unless stated otherwise, a repeated index implies summation over the range of the index. We substitute for $u_{i}$ from Eq. (2) into Eq. (1) to get

$\epsilon=\mathbf{Z}_{\mathbf{i}}\left(\mathrm{X}_{3}\right) \mathbf{L d}_{\mathbf{i}}\left(\mathrm{X}_{1}, \mathrm{X}_{2}\right) \quad(\mathrm{i}=0,1,2,3)$

where

$\epsilon=\left[\epsilon_{11} \in_{22} \in_{33} \quad 2 \epsilon_{23} \quad 2 \epsilon_{13} \quad 2 \epsilon_{12}\right]^{\mathrm{T}}$,

and

$\mathbf{d}_{\mathbf{0}}=\left[\begin{array}{lll}\mathrm{u}_{10} & \mathrm{u}_{20} & \mathrm{u}_{30}\end{array}\right]^{\mathrm{T}}, \mathbf{d}_{\mathbf{1}}=\left[\begin{array}{lll}\mathrm{u}_{11} & \mathrm{u}_{21} & \mathrm{u}_{31}\end{array}\right]^{\mathrm{T}}, \mathbf{d}_{\mathbf{2}}=\left[\begin{array}{lll}\mathrm{u}_{12} & \mathrm{u}_{22} & \mathrm{u}_{32}\end{array}\right]^{\mathrm{T}}$ and $\mathbf{d}_{\mathbf{3}}=\left[\begin{array}{lll}\mathrm{u}_{13} & \mathrm{u}_{23} & \mathrm{u}_{33}\end{array}\right]^{\mathrm{T}}$

are, respectively, vectors of the displacement, the slope, the curvature and the curvature gradient at a point on the laminate mid-surface. The 12-dimensional vector $\mathbf{d}=\left[\mathbf{d}_{\mathbf{0}}, \mathbf{d}_{\mathbf{1}}, \mathbf{d}_{\mathbf{2}}\right.$, $\mathbf{d}_{\mathbf{3}}$ ] is called the vector of generalized displacements at a point on the plate mid-surface. The matrices $\mathbf{Z}_{\mathbf{i}}(\mathrm{i}=0,1,2,3)$ and the operator matrix $\mathbf{L}$ in Eq. (3) are defined in the Appendix A, respectively, by Eqs. (A.1) and (A.2).

As for the components of the strain tensor, components of the Cauchy stress tensor are written as a 6-dimensional (6-D) vector

$\sigma=\left[\begin{array}{llllll}\sigma_{11} & \sigma_{22} & \sigma_{33} & \sigma_{23} & \sigma_{13} & \sigma_{12}\end{array}\right]^{\mathrm{T}}$ 
The constitutive relation (Hooke's law) for a linear elastic material is

$$
\sigma_{\mathrm{ij}}=C_{\mathrm{ijmn}} \in_{\mathrm{mn}}, C_{\mathrm{ijmn}}=C_{\mathrm{mnij}}=C_{\mathrm{jimn}},(\mathrm{i}, \mathrm{j}, \mathrm{m}, \mathrm{n}=1,2,3)
$$

Here $\boldsymbol{C}$ is the fourth-order elasticity tensor having 21 independent components for a general anisotropic material. For an orthotropic, a transversely isotropic and an isotropic material, the independent components of $\boldsymbol{C}$ reduce, respectively, to 9,5 and 2.

With respect to the material principal axes Eq. (5) for an orthotropic material of layer $k$ becomes

$$
\left\{\begin{array}{l}
\sigma_{11}^{k} \\
\sigma_{22}^{k} \\
\sigma_{33}^{k} \\
\sigma_{23}^{k} \\
\sigma_{13}^{k} \\
\sigma_{12}^{k}
\end{array}\right\}=\left[\begin{array}{cccccc}
C_{1111}^{k} & C_{1122}^{k} & C_{1133}^{k} & 0 & 0 & 0 \\
C_{1122}^{k} & C_{2222}^{k} & C_{2233}^{k} & 0 & 0 & 0 \\
C_{1133}^{k} & C_{2233}^{k} & C_{3333}^{k} & 0 & 0 & 0 \\
0 & 0 & 0 & C_{2323}^{k} & 0 & 0 \\
0 & 0 & 0 & 0 & C_{1313}^{k} & 0 \\
0 & 0 & 0 & 0 & 0 & C_{1212}^{k}
\end{array}\right]\left\{\begin{array}{c}
\epsilon_{11}^{k} \\
\epsilon_{22}^{k} \\
\epsilon_{33}^{k} \\
2 \epsilon_{23}^{k} \\
2 \epsilon_{13}^{k} \\
2 \epsilon_{12}^{k}
\end{array}\right\}
$$

where quantities for the $k^{\text {th }}$ layer are indicated by the superscript $k$. In the global coordinate axes $\left(\mathrm{X}_{1}, \mathrm{X}_{2}, \mathrm{X}_{3}\right) C_{\mathrm{ijmn}}$ are computed by using the tensor transformation rules for the stress and the strain tensors, and the 6 x 6 matrix may be fully populated; e.g. see [39]. For the TSNDT, the elastic constants in Eq. (6) are the same as those used in the LET, i.e., they are not modified to satisfy $\in_{33}=0$ as is often done in the CPT.

We use the principle of minimum potential energy, given by Eq. (7), to derive equations governing static deformations of the plate:

$$
\delta \Pi=0
$$

Here, $\delta$ is the variational operator, and $\Pi$ is the potential energy of the plate which in the absence of body forces is given by

$$
\Pi=\frac{1}{2} \sum_{k=1}^{\mathrm{N}} \int_{\Omega^{k}}\left(\epsilon^{k}\right)^{\mathrm{T}} \boldsymbol{\sigma}^{k} \mathrm{~d} \Omega^{k}-\int_{\overline{\mathrm{A}}} \mathbf{u}^{\mathrm{T}} \overline{\mathbf{f}} \mathrm{d} \overline{\mathrm{A}}
$$

in which $\Omega^{k}$ represents the region occupied by the $k^{\text {th }}$ layer, and $\overline{\mathrm{A}}$ is the part of the bounding surface of the plate on which surface traction, $\overline{\mathbf{f}}$, is specified. Points on the remainder of the boundary of the domain, $\Omega$, occupied by the plate have either null tractions 
(i.e., are on a free surface) or have displacements prescribed on them. The work done by reaction forces at points of the boundary where displacements are prescribed is not included in Eq. (8) because there variations in the prescribed displacements are null. Thus equilibrium equations are derived from

$$
\delta \Pi=\sum_{k=1}^{\mathrm{N}} \int_{\Omega^{k}}\left(\delta \epsilon^{k}\right)^{\mathrm{T}} \boldsymbol{\sigma}^{k} \mathrm{~d} \Omega^{k}-\int_{\overline{\mathrm{A}}} \delta \mathbf{u}^{\mathrm{T}} \overline{\mathbf{f}} \mathrm{d} \overline{\mathrm{A}}=0
$$

We substitute in Eq. (9) for $\sigma^{k}$ in terms of $\epsilon^{k}$ from Eq. (6), and substitute for $\epsilon^{k}$ in terms of the generalized displacements defined on the laminate mid-surface from Eq. (3). Also, we substitute for $\mathbf{u}$ in terms of $\mathbf{d}_{\mathbf{i}}(\mathrm{i}=0,1,2,3)$ from Eq. (2). In the resulting expression for $\delta \Pi$, we integrate with respect to $X_{3}$ over the plate thickness to obtain the following integral equation:

$$
\delta \Pi=\int_{0}^{b} \int_{0}^{a} \delta \mathbf{d}_{\mathbf{i}}^{\mathrm{T}} \mathbf{L}^{\mathrm{T}} \mathbf{D}_{\mathbf{i j}} \mathbf{L} \mathbf{d}_{\mathbf{j}} \mathrm{d} \mathbf{X}_{1} \mathrm{~d} \mathbf{X}_{2}-\int_{\overline{\mathrm{A}}} \delta \mathbf{d}_{\mathbf{i}}^{\mathrm{T}} \mathbf{X}_{3}^{\mathrm{i}} \overline{\mathbf{f}} \mathrm{d} \overline{\mathrm{A}}=0 \quad(\mathrm{i}, \mathbf{j}=0,1,2,3)
$$

where $\mathbf{D}_{\mathbf{i j}}=\sum_{k=1}^{\mathrm{N}} \int_{\mathrm{h}_{k}}^{\mathrm{h}_{k+1}} \mathbf{Z}_{\mathbf{i}}^{\mathrm{T}} \mathbf{C}^{k} \mathbf{Z}_{\mathbf{j}} \mathrm{d} \mathbf{X}_{3}$

The mid-surface, $\mathfrak{R}=[0, a] \times[0, b]$, of the plate is discretized into a FE mesh of $\mathrm{N}_{\mathrm{e}}$ disjoint 8-node iso-parametric elements where the region $\mathfrak{R}_{\mathrm{e}}$ occupied by the element e is given by, $\mathfrak{R}_{\mathrm{e}}=\left[\mathrm{X}_{1}^{\mathrm{e}}, \mathrm{X}_{1}^{\mathrm{e}+1}\right] \mathrm{x}\left[\mathrm{X}_{2}^{\mathrm{e}}, \mathrm{X}_{2}^{\mathrm{e}+1}\right]$. Thus $\delta \Pi$ equals the sum of integrals over each element. The 12-dimensional vector $\mathbf{d}$ of generalized displacements at a point in an element is expressed in terms of values of $\mathbf{d}$ at the 8-nodes using the FE basis functions. Thus the total number of unknowns in the problem equals $12 \mathrm{~N}_{\text {node }}$ where $\mathrm{N}_{\text {node }}$ equals the number of nodes. We note that in the FE formulation of the corresponding 3-D problem, the number of unknowns equals $3 \mathrm{~N}_{\text {node }}^{*}$. Since $\mathrm{N}_{\text {node }}^{*}>\mathrm{N}_{\text {node }}$, the total number of unknowns for the TSNDT will be much less than that for the 3-D problem. Following the standard procedure, we obtain from Eq. (10) a system of algebraic equations. Requiring that the resulting equations hold for all choices of $\delta \mathbf{d}_{\mathbf{i}}(\mathrm{i}=0,1,2,3)$ we obtain the following equilibrium equations (see details in Appendix B):

$\mathbf{K U}=\mathbf{F}$ 
In Eq. (11) $\mathbf{K}$ is the global stiffness matrix, $\mathbf{U}$ the global vector of generalized nodal displacements, and $\mathbf{F}$ the global load vector; expressions for these matrices are given as Eq. (B.7) in the Appendix. The vector $\mathbf{F}$ of generalized forces at nodes is work equivalent to surface tractions applied on the top, the bottom, and the edge surfaces of the plate. For a FE mesh of $\mathrm{N}_{\text {node }}$ nodes, before applying essential boundary conditions, the length of vector $\mathbf{U}$ equals $12 \mathrm{~N}_{\text {node }}$ since a node has 12 degrees of freedom.

We consider five types of boundary conditions (BCs) specified at a point on a plate edge. For example, at the edges $\mathrm{X}_{1}=0$ and $a$, the definitions of these BCs in the 3-D LET and their equivalent in terms of variables of the TSNDT are given in Table 1.

Table 1 Nomenclature for boundary conditions specified at $\mathrm{X}_{1}=0$ or $a$

\begin{tabular}{cccc}
\hline Notation & Name & BCs in the 3-D LET & BCs in the TSNDT \\
\hline A & Clamped & $\mathrm{u}_{1}=0, \mathrm{u}_{2}=0, \mathrm{u}_{3}=0$ & $\mathrm{u}_{1 \mathrm{i}}=0, \mathrm{u}_{2 \mathrm{i}}=0, \mathrm{u}_{3 \mathrm{i}}=0$ \\
B & Simply supported & $\sigma_{11}=0, \mathrm{u}_{2}=0, \mathrm{u}_{3}=0$ & $\mathrm{M}_{11}^{\mathrm{i}}=0, \mathrm{u}_{2 \mathrm{i}}=0, \mathrm{u}_{3 \mathrm{i}}=0$ \\
C & - & $\mathrm{u}_{1}=0, \mathrm{u}_{2}=0, \sigma_{13}=0$ & $\mathrm{u}_{1 \mathrm{i}}=0, \mathrm{u}_{2 \mathrm{i}}=0, \mathrm{M}_{13}^{\mathrm{i}}=0$ \\
D & - & $\mathrm{u}_{1}=0, \sigma_{12}=0, \mathrm{u}_{3}=0$ & $\mathrm{u}_{1 \mathrm{i}}=0, \mathrm{M}_{12}^{\mathrm{i}}=0, \mathrm{u}_{3 \mathrm{i}}=0$ \\
F & Traction free & $\sigma_{11}=0, \sigma_{12}=0, \sigma_{13}=0$ & $\mathbf{M}_{11}^{\mathrm{i}}=0, \mathrm{M}_{12}^{\mathrm{i}}=0, \mathrm{M}_{13}^{\mathrm{i}}=0$ \\
\hline
\end{tabular}

In Table 1 the index $i$ takes values $0,1,2$ and 3, and

$\mathbf{M}_{1 \mathrm{n}}^{\mathrm{i}}=\int_{-\mathrm{h} / 2}^{\mathrm{h} / 2} \mathrm{X}_{3}^{\mathrm{i}} \sigma_{1 \mathrm{n}} \mathrm{d} \mathbf{X}_{3}(\mathrm{n}=1,2,3)$

Displacement (or essential) boundary conditions applied at points on a plate edge are satisfied while solving algebraic Eqs. (11).

\section{Numerical Solution of Example Problems}

We analyze static deformations of monolithic and laminated orthotropic plates with values of their material properties with respect to the material principal axes given by either Data set 1 or Data set 2 and compare computed results with those obtained by using the 3-D LET and either analytical techniques or the commercial FE software, ABAQUS, with a uniform $100 \mathrm{x}$ 100 x 10 FE mesh (336,633 DoF) of 8-node brick elements. 
Data set 1:

$\mathrm{E}_{1}=172.5 \mathrm{GPa}, \mathrm{E}_{1} / \mathrm{E}_{2}=25, \mathrm{E}_{3}=\mathrm{E}_{2}, \mathrm{G}_{12}=\mathrm{G}_{13}=0.5 \mathrm{E}_{2}, \mathrm{G}_{23}=0.2 \mathrm{E}_{2}, v_{12}=v_{13}=v_{23}=0.25$

Data set 2:

$\mathrm{E}_{1}=251 \mathrm{GPa}, \mathrm{E}_{2}=48 \mathrm{GPa}, \mathrm{E}_{3}=7.5 \mathrm{GPa}, \mathrm{G}_{12}=13.6 \mathrm{GPa}, \mathrm{G}_{13}=12 \mathrm{GPa}, \mathrm{G}_{23}=4.7 \mathrm{GPa}$, $v_{12}=0.036, v_{13}=0.25, v_{23}=0.171$

Here E denotes Young's modulus, $G$ the shear modulus and $v$ Poisson's ratio. When discussing results below we use the more common notation and replace $X_{1}, X_{2}$ and $X_{3}$ by $\mathrm{x}, \mathrm{y}$ and $\mathrm{z}$, respectively, and $\mathrm{u}_{1}, \mathrm{u}_{2}$ and $\mathrm{u}_{3}$ by $\mathrm{u}, \mathrm{v}$ and $\mathrm{w}$, respectively.

The in-plane stresses $\left(\sigma_{\mathrm{xx}}, \sigma_{\mathrm{yy}}, \sigma_{\mathrm{xy}}\right)$ are obtained directly from the constitutive relations and displacements found using the plate theory. Through-the-thickness variations of the transverse shear $\left(\sigma_{\mathrm{xz}}, \sigma_{\mathrm{yz}}\right)$ and the transverse normal $\left(\sigma_{\mathrm{zz}}\right)$ stresses have been computed by using the constitutive relation for monolithic plates and by employing the stress-recovery scheme (SRS) for laminated plates; these are denoted by C and SRS, respectively, in the plots. In the SRS, the three equilibrium equations are integrated with respect to $\mathrm{z}$ starting from the bottom face with surface tractions prescribed there as "initial conditions". At interfaces between two adjoining layers, the traction and the displacement continuity conditions are satisfied; the former because we have used the single layer theory and the latter during integration of equilibrium equations with respect to $\mathrm{z}$. The difference between surface tractions thus computed and the applied surface tractions on the top surface is an indicator of the error in the numerical solution of the problem. In the SRS scheme, the spatial gradients of the in-plane stresses are found first by computing the stresses at the $3 \times 3$ quadrature points in each FE on the mid-surface, fitting a complete quadratic polynomial to the stress values at the 9-points by the least squares method, and then differentiating the polynomial function with respect to $\mathrm{x}$ and $\mathrm{y}$. We note that for a quadratic serendipity element, Barlow [52] found the optimal stress points coincide with the $2 \times 2$ Gauss points for the Lagrange quadratic element. 


\subsection{Convergence of the solution with $\mathrm{FE}$ mesh refinement}

For six uniform $\mathrm{n} x \mathrm{~m}$ FE meshes with $\mathrm{n}$ and $\mathrm{m}$ elements along the $\mathrm{x}$ - and the y-axes, respectively, we have listed in Table 2(a) values of the non-dimensional deflection, $\overline{\mathrm{w}}(0.5 a, 0.5 b, 0)$, at the centroid of the mid-surface, the axial stress, $\bar{\sigma}_{\mathrm{xx}}(0.5 a, 0.5 b, 0.5 \mathrm{~h})$, at the centroid of the top surface and the transverse shear stress, $\bar{\sigma}_{\mathrm{xz}}(0.1 a, 0.5 b, 0)$, near the edge $\mathrm{x}=0$ on the mid-surface of a $0^{\circ} / 90^{\circ} / 0^{\circ}$ laminated square plate with all edges clamped and loaded only by a uniformly distributed tensile traction, $\mathrm{q}_{0}$, on the top surface. The corresponding results for the plate with simply supported BCs on edges $\mathrm{y}=0$ and $b$ and the BC classified as D in Table 1 on the other two edges are presented in Table 2(b). We note that the transverse shear stress is computed by using the SRS. The displacement and stresses are non-dimensionalized as: $\overline{\mathrm{w}}=\mathrm{w}\left(\mathrm{h}^{3} / b^{4}\right) \mathrm{E}_{2} / \mathrm{q}_{0}$ and $\left\{\bar{\sigma}_{\mathrm{xx}}, \bar{\sigma}_{\mathrm{xz}}\right\}=\left\{\sigma_{\mathrm{xx}}, \sigma_{\mathrm{xz}}\right\} / \mathrm{q}_{0}$. The lamination scheme denoted by $\alpha_{1} / \alpha_{2} / \ldots / \alpha_{N}$ for a laminate having $N$ layers with layers 1 and $\mathrm{N}$ being the bottom and the top layers, respectively, indicates that fibers in the $\mathrm{k}^{\text {th }}$ layer are oriented at an angle $\alpha_{k}$ measured counter clockwise from the $\mathrm{x}$ - axis. The values of elastic constants for orthotropic material of the plate are given by Data set 1 and values assigned to other parameters are $\mathrm{h}=10 \mathrm{~mm}, a / \mathrm{h}=10, a=b$, and $\mathrm{q}_{0}=10 \mathrm{MPa}$. Numbers in the column "Diff." denote the change in the value from that obtained with the immediate previous FE mesh.

Table 2(a) Convergence of solutions for a clamped $0^{\circ} / 90 \% 0^{\circ}$ laminated plate $(a / \mathrm{h}=10, a=b)$

\begin{tabular}{ccccccc}
\hline Mesh & $\overline{\mathrm{w}}(0.5 a, 0.5 b, 0) \times 100$ & $\%$ Diff. & $\bar{\sigma}_{\mathrm{xx}}(0.5 a, 0.5 b, 0.5 \mathrm{~h})$ & $\%$ Diff. & $\bar{\sigma}_{\mathrm{xz}}(0.1 a, 0.5 b, 0)$ & $\%$ Diff. \\
\hline $15 \times 15$ & 0.5212 & - & 33.076 & - & 4.364 & - \\
$17 \times 17$ & 0.5213 & 0.02 & 33.100 & 0.07 & 4.349 & -0.34 \\
$19 \times 19$ & 0.5213 & 0.00 & 33.116 & 0.05 & 4.313 & -0.83 \\
$21 \times 21$ & 0.5213 & 0.00 & 33.128 & 0.04 & 4.364 & 1.17 \\
$23 \times 23$ & 0.5214 & 0.02 & 33.136 & 0.02 & 4.388 & 0.55 \\
$25 \times 25$ & 0.5214 & 0.00 & 33.145 & 0.03 & 4.391 & 0.07 \\
\hline
\end{tabular}

Table 2(b) Convergence of solutions for a $0^{\circ} / 90^{\circ} / 0^{\circ}$ laminated plate $(a / \mathrm{h}=10, a=b)$ with simply supported BCs on edges $\mathrm{y}=0, \mathrm{~b}$ and the $\mathrm{BC}$ type $\mathrm{D}$ on the other two edges

\begin{tabular}{ccccccc}
\hline Mesh & $\overline{\mathrm{w}}(0.5 a, 0.5 b, 0) \times 100$ & \%Diff. & $\bar{\sigma}_{\mathrm{xx}}(0.5 a, 0.5 b, 0.5 \mathrm{~h})$ & \%Diff. & $\bar{\sigma}_{\mathrm{xz}}(0.1 a, 0.5 b, 0)$ & $\%$ Diff. \\
\hline $15 \times 15$ & 0.6026 & - & 38.802 & - & 4.968 & - \\
$17 \times 17$ & 0.6026 & 0.00 & 38.829 & 0.07 & 4.942 & -0.53
\end{tabular}




\begin{tabular}{lcccccc}
$19 \times 19$ & 0.6026 & 0.00 & 38.848 & 0.05 & 4.895 & -0.96 \\
$21 \times 21$ & 0.6026 & 0.00 & 38.862 & 0.04 & 4.979 & 1.69 \\
$23 \times 23$ & 0.6026 & 0.00 & 38.872 & 0.03 & 4.998 & 0.38 \\
$25 \times 25$ & 0.6026 & 0.00 & 38.881 & 0.02 & 4.995 & -0.06 \\
\hline
\end{tabular}

It is clear that for both problems the $23 \times 23 \mathrm{FE}$ mesh gives converged values of the computed quantities since the change in their values in going from the $23 \times 23$ to the $25 \times 25$ FE mesh is rather minuscule. At least for these two problems studied, the deflection and the in-plane axial stress converge faster than the transverse shear stress with the mesh refinement and the convergence rate does not depend much upon the BCs. Note that we have used the ESL theory for the laminated plate. Unless mentioned otherwise, we will use the $25 \times 25$ uniform FE mesh which corresponds to 23,712 nodal degrees of freedom (DoF) to obtain numerical solutions using the TSNDT for the example problems studied.

We now study seven example problems for monolithic and laminated plates subjected to a variety of traction boundary conditions on their major surfaces and five different BCs defined in Table 1 on their edges in order to test the applicability of the TSNDT and the SRS to evaluate stresses at points near the plate edges for a wide range of problems.

\subsection{Normal uniform surface traction on isotropic plate}

\subsubsection{Transverse normal and shear stresses from the SRS}

We analyze deformations of a clamped square isotropic plate with $\mathrm{h}=1 \mathrm{~cm}, a / \mathrm{h}=10$, values of material parameters given by $\mathrm{E}=210 \mathrm{GPa}, \nu=0.3$, and a uniformly distributed normal tensile traction, $\mathrm{q}_{0}=10 \mathrm{MPa}$, applied only on the top surface. In Fig. 2(a) we have displayed through-the-thickness distributions of the axial stress, $\sigma_{\mathrm{xx}}$, and the transverse normal stress, $\sigma_{\mathrm{zz}}$, along the transverse normal passing through the centroid of the plate and computed using the constitutive relations and the TSNDT displacement field, and the FE solution based on the 3-D LET. The two sets of results agree well with each other. For the TSNDT, the transverse normal stress obtained from the constitutive relation does not exhibit the "boundary layer" phenomenon near the top and the bottom surfaces of the plate. Furthermore, 
the computed value of $\sigma_{\mathrm{zz}}$ differs from the normal traction applied at the point $(0.5 a, 0.5 b$, $0.5 \mathrm{~h}$ ) by $22 \%$. However, this difference is reduced to $0.84 \%$ and the "boundary layer" effect is accurately captured when the transverse normal stress is computed using the SRS. We note that through-the-thickness distribution of $\sigma_{\mathrm{zz}}$ computed from the constitutive relation and the TSNDT displacement field is quite different from that found using the 3-D LET. The difference between the deflection, $w$, and the axial stress, $\sigma_{\mathrm{xx}}$, at the centroid of the top surface obtained with the uniform $100 \times 100 \times 10$ and the uniform $150 \times 150 \times 15$ FE meshes $(1,094,448 \mathrm{DoF})$ is $0.04 \%$ and $0.6 \%$, respectively. In Fig. 2(b) we have displayed throughthe-thickness variation of the transverse shear stress, $\sigma_{\mathrm{xz}}$, along the transverse normal passing through the point $(0.1 a, 0.5 b, 0)$ near the edge $\mathrm{x}=0$. It is evident that results from the TSNDT agree well with those from the 3-D LET solution both when the stress is obtained from the constitutive relation and the SRS with the maximum difference of $1.52 \%$ at $\mathrm{z}=0$.

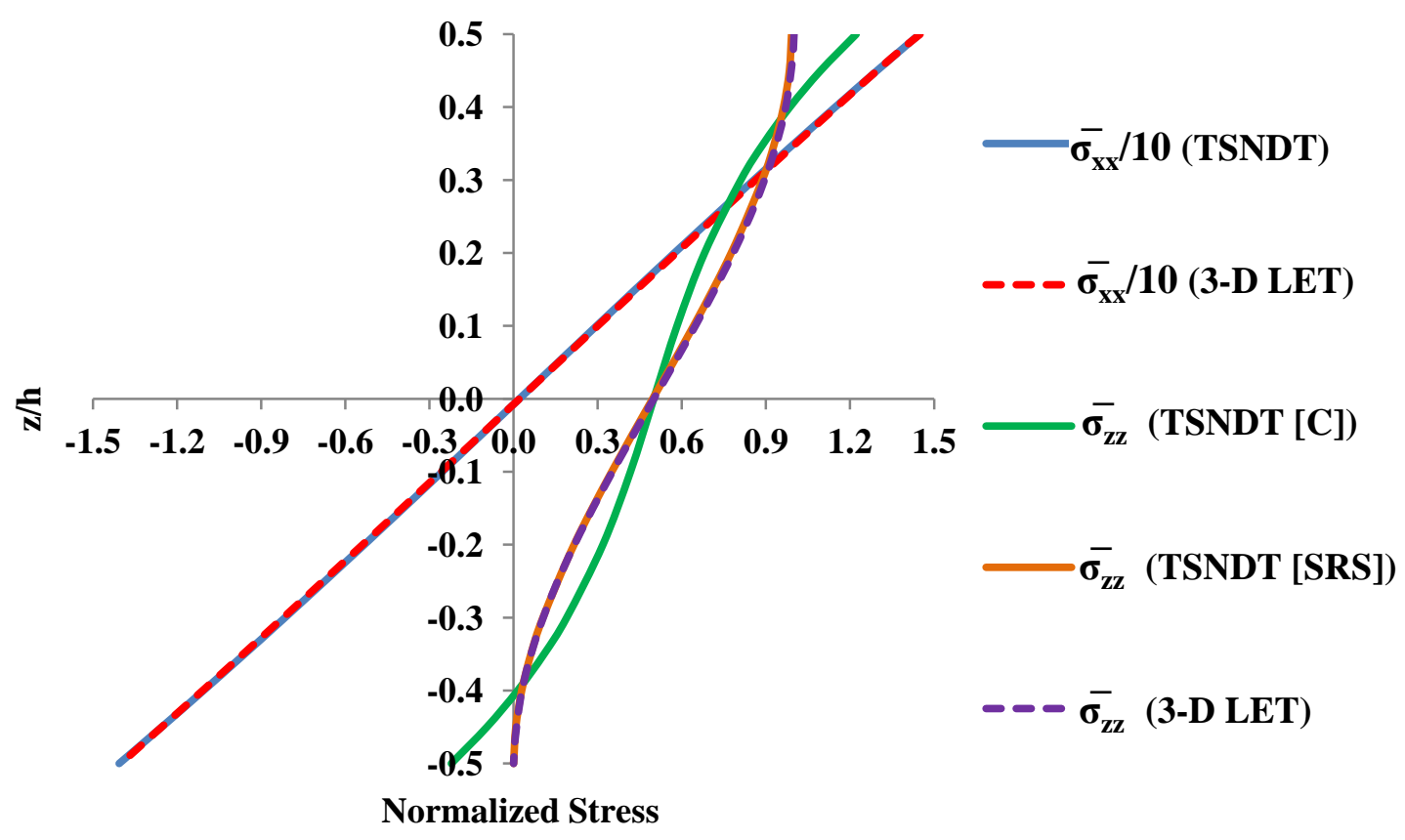

(a) 


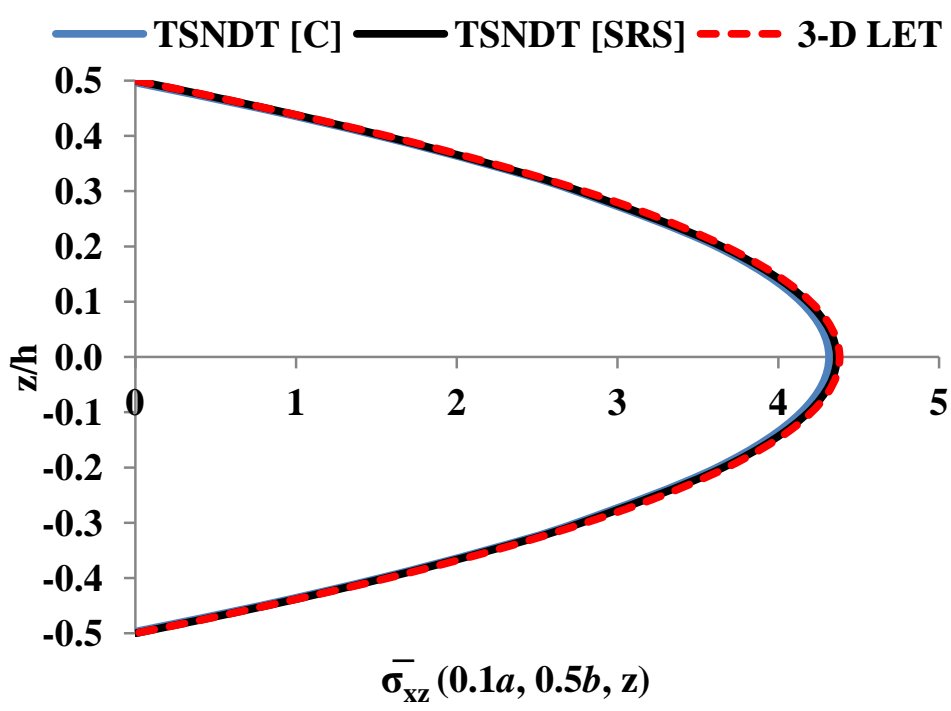

(b)

Fig. 2 For a clamped isotropic plate subjected to a uniform normal traction on its top surface, through-the-thickness distribution of (a) $\bar{\sigma}_{\mathrm{xx}}(0.5 a, 0.5 b, \mathrm{z})$ and $\bar{\sigma}_{\mathrm{zz}}(0.5 a, 0.5 b, \mathrm{z})$, and (b) $\bar{\sigma}_{\mathrm{xz}}(0.1 a, 0.5 b, \mathrm{z})$. Stresses are normalized by $\mathrm{q}_{0}$.

We note that for a very thick (length/thickness $=2$ ) cantilever beam Batra and Vidoli [12] found that a $7^{\text {th }}$ order shear and normal deformable plate theory derived from the mixed variational principle gave accurate values of stresses including the boundary layer phenomenon without using the SRS.

\subsubsection{Effect of plate aspect ratio on the order of plate theory}

In order to ascertain for what aspect ratios of the plate, the TSNDT gives accurate values of stresses, we have studied deformations of the square plate for $a / \mathrm{h}=10,5,3.33,2.5$ and 2. In Table $3 a, b(4 a, b)$ we have listed values of the non-dimensional deflection at the centroid of the mid-surface, $\overline{\mathrm{w}}=\mathrm{w}(0.5 a, 0.5 b, 0)\left(\mathrm{h}^{3} / b^{4}\right) \mathrm{E} / 12 \mathrm{q}_{0}\left(1-\mathrm{v}^{2}\right)$ and the axial stress, $\bar{\sigma}_{\mathrm{xx}}=\sigma_{\mathrm{xx}}(0.5 a, 0.5 b, 0.5 \mathrm{~h})(a / \mathrm{h})^{2} / \mathrm{q}_{0}$ at the centroid of the top surface of the clamped (simply supported) plate predicted by the TSNDT and computed with a $6^{\text {th }}$ order shear and normal deformable plate theory (SSNDT) [15] using two local meshless Petrov-Galerkin formulations, namely MLPG1 and MLPG5, along with their differences with respect to the 3D LET solutions [15]. 
Table 3(a) Non-dimensional deflection, $\overline{\mathrm{w}}$, of the centroid of the clamped plate

\begin{tabular}{cccccccc}
\hline$a / \mathrm{h}$ & 3-D LET [15] & $\begin{array}{c}\text { SSNDT + } \\
\text { MLPG1 [15] }\end{array}$ & \%Diff. & $\begin{array}{c}\text { SSNDT + } \\
\text { MLPG5 [15] }\end{array}$ & \%Diff. & TSNDT & $\%$ Diff. \\
\hline 10 & 0.1486 & 0.1468 & 1.21 & 0.1476 & 0.67 & 0.1486 & 0.03 \\
5 & 0.2124 & 0.2112 & 0.56 & 0.2103 & 0.99 & 0.2119 & 0.24 \\
3.33 & 0.3129 & 0.3119 & 0.32 & 0.3064 & 2.08 & 0.3116 & 0.43 \\
2.5 & 0.4471 & 0.4470 & 0.02 & 0.4408 & 1.41 & 0.4451 & 0.46 \\
2 & 0.6114 & 0.6125 & -0.18 & 0.6050 & 1.05 & 0.6090 & 0.40 \\
\hline
\end{tabular}

Table 3(b) Non-dimensional stress, $\bar{\sigma}_{x x}$, at the centroid of the top surface of the clamped plate

\begin{tabular}{cccccccc}
\hline$a / \mathrm{h}$ & 3-D LET [15] & $\begin{array}{c}\text { SSNDT + } \\
\text { MLPG1 [15] }\end{array}$ & \%Diff. & $\begin{array}{c}\text { SSNDT + } \\
\text { MLPG5 [15] }\end{array}$ & \%Diff. & TSNDT & \%Diff. \\
\hline 10 & 0.1440 & 0.1432 & 0.56 & 0.1450 & -0.69 & 0.1448 & -0.56 \\
5 & 0.1613 & 0.1617 & -0.25 & 0.1589 & 1.49 & 0.1658 & -2.76 \\
3.33 & 0.1877 & 0.1895 & -0.96 & 0.1836 & 2.18 & 0.1979 & -5.43 \\
2.5 & 0.2235 & 0.2274 & -1.74 & 0.2224 & 0.49 & 0.2419 & -8.25 \\
2 & 0.2725 & 0.2887 & -5.94 & 0.2725 & 0.00 & 0.3021 & -10.86 \\
\hline
\end{tabular}

Table 4(a) Non-dimensional deflection, $\overline{\mathrm{w}}$, of the centroid of the simply supported plate

\begin{tabular}{cccccccc}
\hline$a / \mathrm{h}$ & 3-D LET [15] & $\begin{array}{c}\text { SSNDT + } \\
\text { MLPG1 [15] }\end{array}$ & \%Diff. & $\begin{array}{c}\text { SSNDT + } \\
\text { MLPG5 [15] }\end{array}$ & \%Diff. & TSNDT & $\%$ Diff. \\
\hline 10 & 0.4249 & 0.4220 & 0.68 & 0.4275 & -0.61 & 0.4249 & 0.00 \\
5 & 0.4803 & 0.4798 & 0.10 & 0.4793 & 0.21 & 0.4804 & -0.03 \\
3.33 & 0.5710 & 0.5717 & -0.12 & 0.5589 & 2.12 & 0.5716 & -0.11 \\
2.5 & 0.6952 & 0.6967 & -0.22 & 0.6807 & 2.09 & 0.6965 & -0.19 \\
2 & 0.8487 & 0.8511 & -0.28 & 0.8304 & 2.16 & 0.8522 & -0.41 \\
\hline
\end{tabular}

Table 4(b) Non-dimensional stress, $\bar{\sigma}_{\mathrm{xx}}$, at the centroid of the top surface of the simply supported plate

\begin{tabular}{cccccccc}
\hline$a / \mathrm{h}$ & \multirow{2}{*}{ 3-D LET [15] } & $\begin{array}{c}\text { SSNDT + } \\
\text { MLPG1 [15] }\end{array}$ & \%Diff. & $\begin{array}{c}\text { SSNDT + } \\
\text { MLPG5 [15] }\end{array}$ & \%Diff. & TSNDT & $\%$ Diff. \\
\hline 10 & 0.2900 & 0.2887 & 0.45 & 0.2920 & -0.69 & 0.2910 & -0.35 \\
5 & 0.2976 & 0.2984 & -0.27 & 0.3020 & -1.48 & 0.3023 & -1.59 \\
3.33 & 0.3099 & 0.3129 & -0.97 & 0.3110 & -0.35 & 0.3209 & -3.56 \\
2.5 & 0.3283 & 0.3333 & -1.52 & 0.3286 & -0.09 & 0.3472 & -5.75 \\
2 & 0.3568 & 0.3640 & -2.02 & 0.3692 & -3.48 & 0.3877 & -8.65 \\
\hline
\end{tabular}

It is found that for $a / \mathrm{h} \geq 5$, the TSNDT solutions agree well with those from the SSNDT and from the 3-D LET. For thicker plates, stresses from the SSNDT are in better agreement with those from the 3-D LET than those from the TSNDT. The maximum difference between values of axial stress, $\bar{\sigma}_{\mathrm{xx}}=\sigma_{\mathrm{xx}}(0.5 a, 0.5 b, 0.5 \mathrm{~h})(a / \mathrm{h})^{2} / \mathrm{q}_{0}$, obtained from the TSNDT and 
the 3 -D LET is about $11 \%(9 \%)$ when $a / \mathrm{h}=2$ and all edges of the plate are clamped (simply supported).

\subsubsection{Effect of plate aspect ratio on strain energies of different modes of deformation}

We now study the effect of the plate aspect ratio on strain energies of different modes of deformation of the clamped plate with $a=10 \mathrm{~cm}$. The total strain energy, W, of deformation is given by

$$
\mathrm{W}=\sum_{\mathrm{i}=1}^{6} \mathrm{~W}_{\mathrm{i}}
$$

where

$$
\begin{aligned}
& \mathrm{W}_{1}=\frac{1}{2} \sum_{k=1}^{\mathrm{N}} \int_{\Omega^{k}} \sigma_{\mathrm{xx}}^{k} \in_{\mathrm{xx}}^{k} \mathrm{~d} \Omega^{k}, \mathrm{~W}_{2}=\frac{1}{2} \sum_{k=1}^{\mathrm{N}} \int_{\Omega^{k}} \sigma_{\mathrm{yy}}^{k} \in_{\mathrm{yy}}^{k} \mathrm{~d} \Omega^{k}, \mathrm{~W}_{3}=\sum_{k=1}^{\mathrm{N}} \int_{\Omega^{k}} \sigma_{\mathrm{xy}}^{k} \in_{\mathrm{xy}}^{k} \mathrm{~d} \Omega^{k}, \\
& \mathrm{~W}_{4}=\sum_{k=1}^{\mathrm{N}} \int_{\Omega^{k}} \sigma_{\mathrm{xz}}^{k} \in_{\mathrm{xz}}^{k} \mathrm{~d} \Omega^{k}, \mathrm{~W}_{5}=\sum_{k=1}^{\mathrm{N}} \int_{\Omega^{k}} \sigma_{\mathrm{yz}}^{k} \in_{\mathrm{yz}}^{k} \mathrm{~d} \Omega^{k}, \mathrm{~W}_{6}=\frac{1}{2} \sum_{k=1}^{\mathrm{N}} \int_{\Omega^{k}} \sigma_{\mathrm{zz}}^{k} \in_{\mathrm{zz}}^{k} \mathrm{~d} \Omega^{k} .
\end{aligned}
$$

Thus $\mathrm{W}_{1}, \mathrm{~W}_{2}$ and $\mathrm{W}_{3}$ equal strain energies of deformation due to in-plane deformations, and $\mathrm{W}_{4}, \mathrm{~W}_{5}$ and $\mathrm{W}_{6}$ due to transverse deformations.

We first verify the computation of the strain energy for a thick square plate with $a / \mathrm{h}=5$ and $a$ $=10 \mathrm{~cm}$ by comparing its value with that of the work done by external forces; both should be equal for a static elastic problem. The strain energy of deformation, $\mathrm{W}$, of the plate is found to be 257.63 and $263.25 \mathrm{~mJ}$ when the transverse stresses are obtained from the constitutive relations and the SRS, respectively. For stresses obtained using the SRS, the corresponding strain tensor is computed by pre-multiplying the stress tensor with the compliance matrix; and $\mathrm{W}_{4}, \mathrm{~W}_{5}$ and $\mathrm{W}_{6}$ are evaluated using the recovered stresses and strains. The work done by the external forces equals $263.09 \mathrm{~mJ}$. Thus, the error in computation of the strain energy is $2.08 \%$ and $-0.06 \%$ when the transverse stresses are obtained from the constitutive relations and the SRS, respectively. This provides another check on the accuracy of the computed results.

We have compared in Table 5 each component of the strain energy computed with the TSNDT solution with that from the 3-D LET solution. The energies computed using stresses obtained from constitutive relations and the SRS, respectively, are displayed in columns 
"TSNDT (C)" and "TSNDT (SRS)", and their differences with respect to the 3-D LET solution are listed in columns "Diff.". The strain energies of deformation predicted by the TSNDT match well with those from the 3-D LET solution. The maximum difference between the results from the two theories is about $11 \%$ for the strain energy of transverse normal deformation. However, its contribution to the total strain energy is very small as compared to that of other deformation modes. We also note that the strain energies of transverse shear deformation are more accurately captured when the SRS is used, the strain energy due to transverse shear stresses is about $12 \%$ more than that due to in-plane axial stresses and the SRS does not provide much better value of $\mathrm{W}_{6}$ than that given by the TSNDT (C).

Table 5 Components of strain energy of deformation $(\mathrm{mJ})$ of the plate

\begin{tabular}{cccccc}
\hline Component & 3-D LET & TSNDT $(\mathrm{C})$ & \%Diff. & TSNDT (SRS) & \%Diff. \\
\hline $\mathrm{W}_{1}, \mathrm{~W}_{2}$ & 56.50 & 55.61 & 1.57 & - & - \\
$\mathrm{W}_{3}$ & 14.41 & 14.35 & 0.44 & - & - \\
$\mathrm{W}_{4}, \mathrm{~W}_{5}$ & 63.96 & 62.35 & 2.51 & 64.33 & -0.58 \\
$\mathrm{~W}_{6}$ & 8.29 & 7.36 & 11.27 & 9.01 & -8.71 \\
Total, $\mathrm{W}$ & 263.62 & 257.63 & 2.27 & 263.25 & 0.14 \\
\hline
\end{tabular}

In Fig. 3 we have illustrated for a plate with $a=b=10 \mathrm{~cm}$ and subjected to the uniform traction, $\mathrm{q}_{0}$, on its top surface only, the effect of the aspect ratio on strain energies of bending $\left(\mathrm{W}_{1}+\mathrm{W}_{2}+\mathrm{W}_{3}\right)$, transverse shear $\left(\mathrm{W}_{4}+\mathrm{W}_{5}\right)$ and transverse normal $\left(\mathrm{W}_{6}\right)$ deformations. It is found that with an increase in the aspect ratio, the strain energy of the bending deformation increases and that of the transverse shear and the transverse normal deformations decreases. The strain energies of transverse deformation have been computed using the SRS. For $a / \mathrm{h}=$ 5 , the transverse shear and the transverse normal deformation modes account for $48.9 \%$ and $3.4 \%$ of the total strain energy, respectively. However, for $a / \mathrm{h}>30$, the transverse shear deformations become negligible and the bending deformations dominate. 


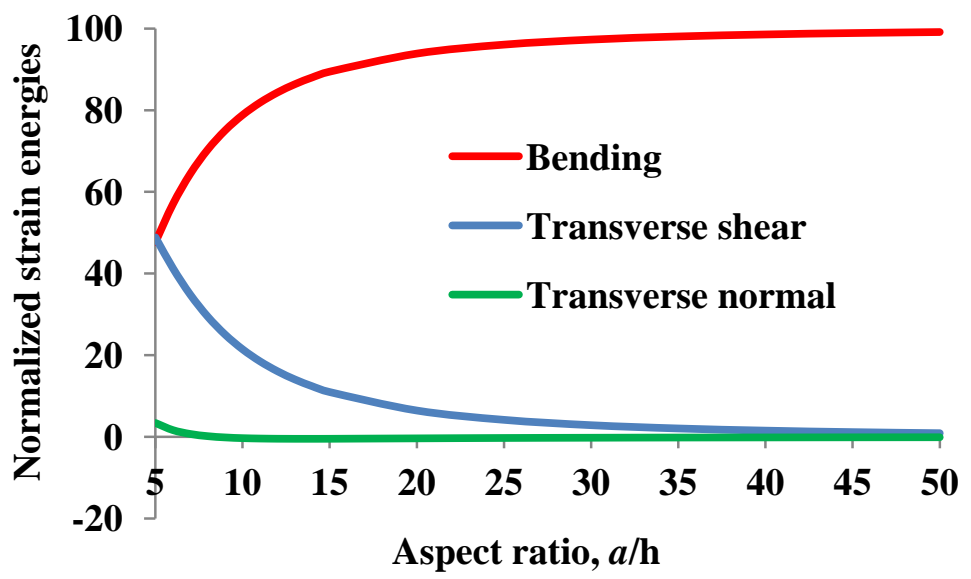

Fig. 3 Variation with the aspect ratio of the normalized values of the strain energies of deformation due to bending, transverse shear and transverse normal deformations for a monolithic clamped plate subjected to uniformly distributed tensile normal traction on the top surface

\subsection{Equal and opposite uniform tangential tractions applied on the top and the bottom surfaces of a $0^{\circ}$ composite plate}

\subsubsection{Effect of plate aspect ratio on strain energies of different modes of deformation}

We study deformations of an orthotropic square plate clamped at the left edge with the remaining three edges traction free, and having $\mathrm{h}=1 \mathrm{~cm}, a / \mathrm{h}=10$, values of material parameters listed in Data set 2, and equal and opposite uniform tangential tractions applied on the top and the bottom surfaces. The inset in Fig. 4 schematically illustrates the problem studied which is similar to the beam problem studied by Batra and Vidoli [12] who used a variable order shear and normal deformable theory. As depicted in Fig. 4, the deformed shapes of the section $\mathrm{y}=b / 2$ predicted by the TSNDT and the 3-D FEM are in excellent agreement with each other. The magnitude of displacement at points $\mathrm{P}$ and $\mathrm{Q}$ is found to be $1.62 \mathrm{~mm}$. 


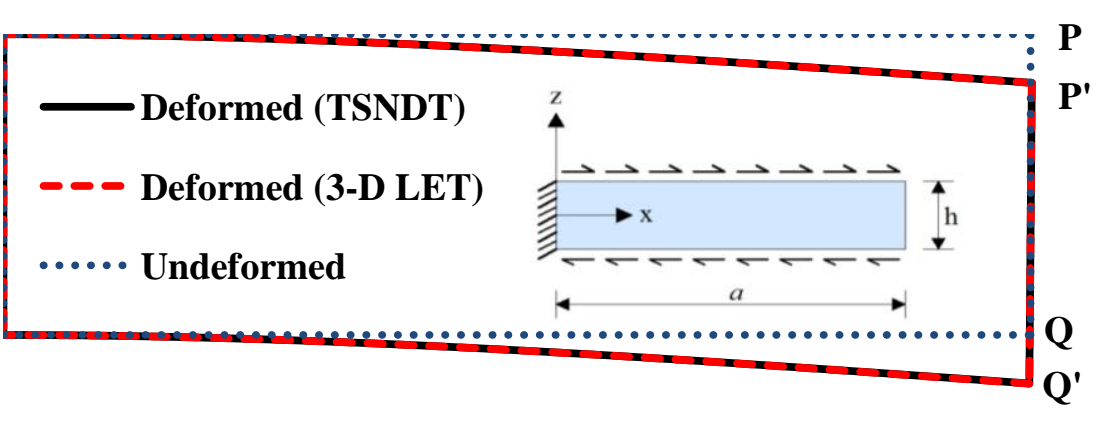

(a)

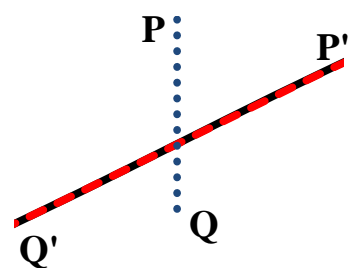

(b)

Fig. 4 For an orthotropic plate subjected to equal and opposite uniform tangential tractions on the top and the bottom surfaces (a) deformed shapes of the section $\mathrm{y}=b / 2$ computed using the TSNDT and the 3-D LET, and (b) an enlarged view on a scale 500 times that in Fig. (a) of the deformation of the segment PQ. The deformed shape P' $Q$ ' of PQ is a polynomial of degree 3.

Figures 5(a) and 5(b) depict through-the-thickness distributions of the axial stress, $\sigma_{\mathrm{xx}}$, and the transverse shear stress, $\sigma_{\mathrm{xz}}$, at various locations near and away from the edges $\mathrm{x}=0$ and a. Values of $\sigma_{\mathrm{xx}}$ obtained using the TSNDT match well with those found by analyzing 3-D deformations with $2 \%$ maximum difference between them at $(0.93 a, 0.5 b, 0)$. The transverse shear stress, $\sigma_{\mathrm{xz}}$, computed from the constitutive relation differs from the corresponding 3-D LET solution by as much as $44.7 \%$ at points on the line defined by $\mathrm{x}=0.07 a, \mathrm{y}=0.5 b$ near the clamped edge $\mathrm{x}=0$ and its value at points of intersection of this line with the two major surfaces differs from the applied tangential traction by $16.4 \%$. These differences reduce to $3.5 \%$ and $10.8 \%$ for points on the line $\mathrm{x}=0.93 a, \mathrm{y}=0.5 b$ near the traction free edge $\mathrm{x}=a$. However, when $\sigma_{\mathrm{xz}}$ is computed using the SRS the tangential traction boundary conditions on the top and the bottom surfaces are exactly satisfied and the maximum differences between the two values from the TSNDT and the 3-D LET solutions reduce to $7.9 \%$ and $1.4 \%$ along the transverse normals passing through the points $(0.07 a, 0.5 b, 0)$ and $(0.93 a, 0.5 b, 0)$, respectively; these maximum differences occur at points on the plate midsurface. Thus the transverse shear stress at a point near the clamped edge is less accurate than that at the corresponding point near the free edge even when the SRS is employed. 


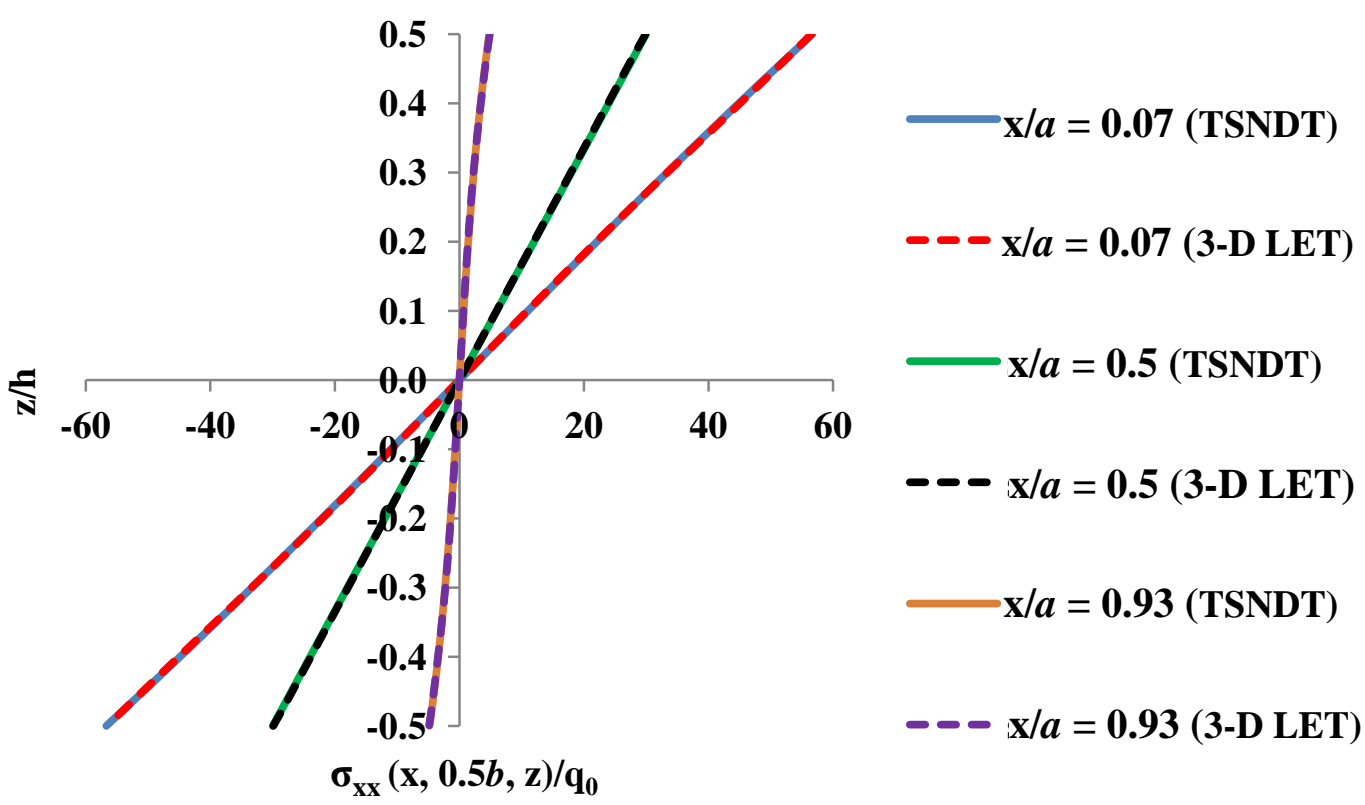

(a)

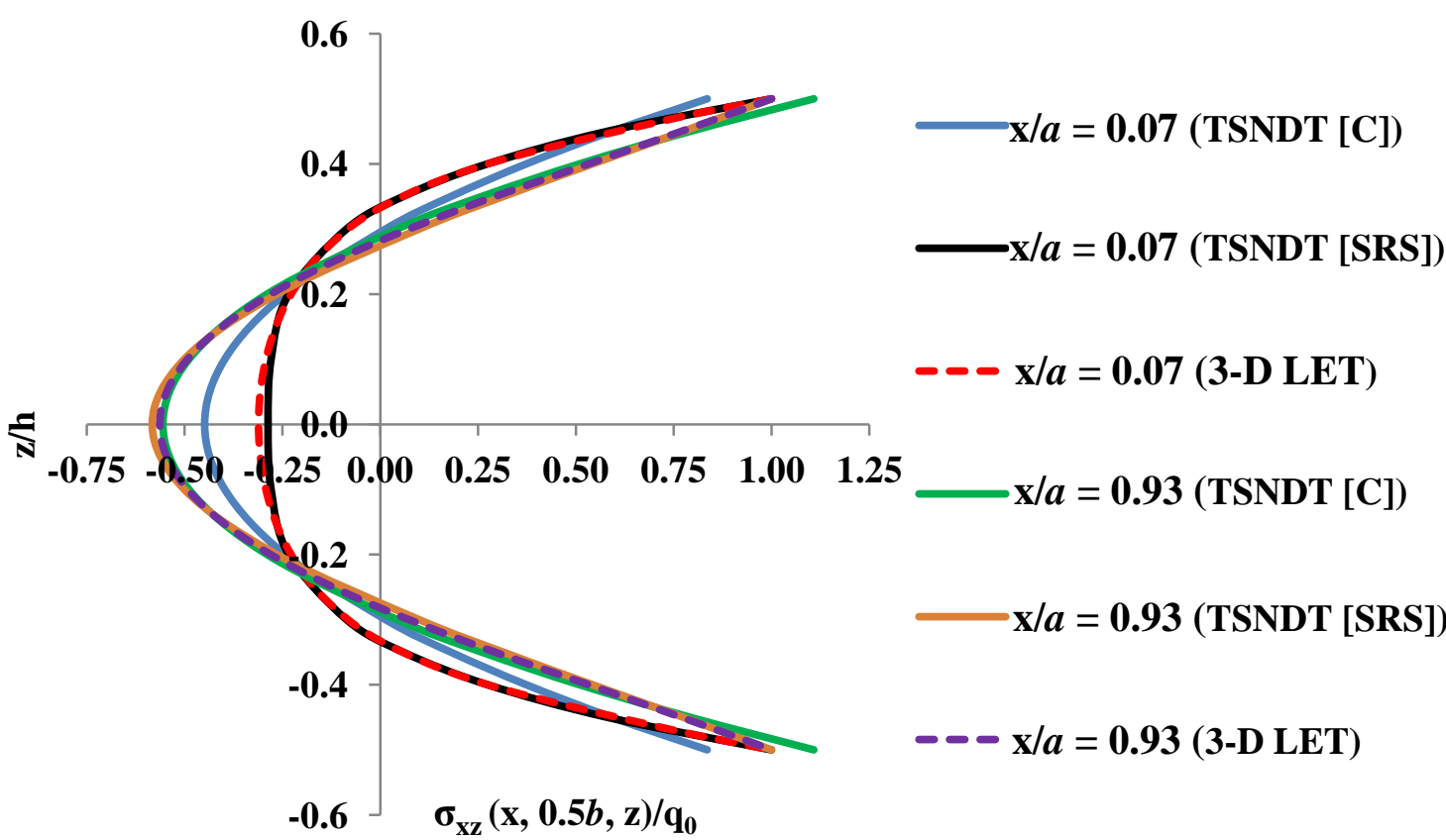

(b)

Fig. 5 For the cantilever orthotropic plate subjected to equal and opposite uniform tangential tractions on the top and the bottom surfaces, through-the-thickness distributions of (a) $\sigma_{\mathrm{xx}}(\mathrm{x}, 0.5 b, \mathrm{z})$ for $\mathrm{x} / a=0.07,0.5$ and 0.93 , and $\sigma_{\mathrm{xz}}(\mathrm{x}, 0.5 b, \mathrm{z})$ for $\mathrm{x} / a=0.07$ and 0.93 . 


\subsubsection{Effect of plate aspect ratio on strain energies of different modes of deformation}

We first analyze plane strain deformations of the plate by neglecting deformations in the ydirection (that is equivalent to studying deformations of a beam) and compare in Fig. 6(a) the strain energies of different deformation modes with the corresponding results based on a $7^{\text {th }}$ order shear and normal deformable plate theory reported by Batra and Vidoli [12]. It is evident that the TSNDT with the SRS accurately predicts strain energies due to different deformation modes for the problem studied. The difference in the strain energies due to bending and shear modes for $a / \mathrm{h}=2$ computed from the TSNDT and that predicted by the $7^{\text {th }}$ order theory is $1.85 \%$ and $5.58 \%$. The contribution from the transverse shear deformation mode to the total strain energy of the beam is about $25 \%$ for $a / \mathrm{h}=2$ and becomes negligible for $a / h=20$. In Fig. 6(b) we have exhibited similar results for the plate problem described in Fig. 4 inset with $a=b=10 \mathrm{~cm}$ and compared with the corresponding results for the beam from the $7^{\text {th }}$ order theory. We note that in Fig. 6(b) the strain energies of transverse shear and normal modes and the bending modes are plotted, respectively, on the left and the right vertical axes. For $a / \mathrm{h}=4$, the transverse shear deformation modes contribute only $5.5 \%$ to the total strain energy of the plate and its contribution further decreases with an increase in the aspect ratio. The strain energy due to transverse normal deformations is negligible because of uniform tangential tractions applied on the two major surfaces. It is interesting to note that for $a / \mathrm{h}=5$, the strain energy due to transverse shear deformations equals about $3.7 \%$ and $12 \%$ of the total strain energy of deformations for the plate and the beam, respectively. Thus results for the beam are not necessarily indicative of those for the plate under similar boundary conditions and loads. 


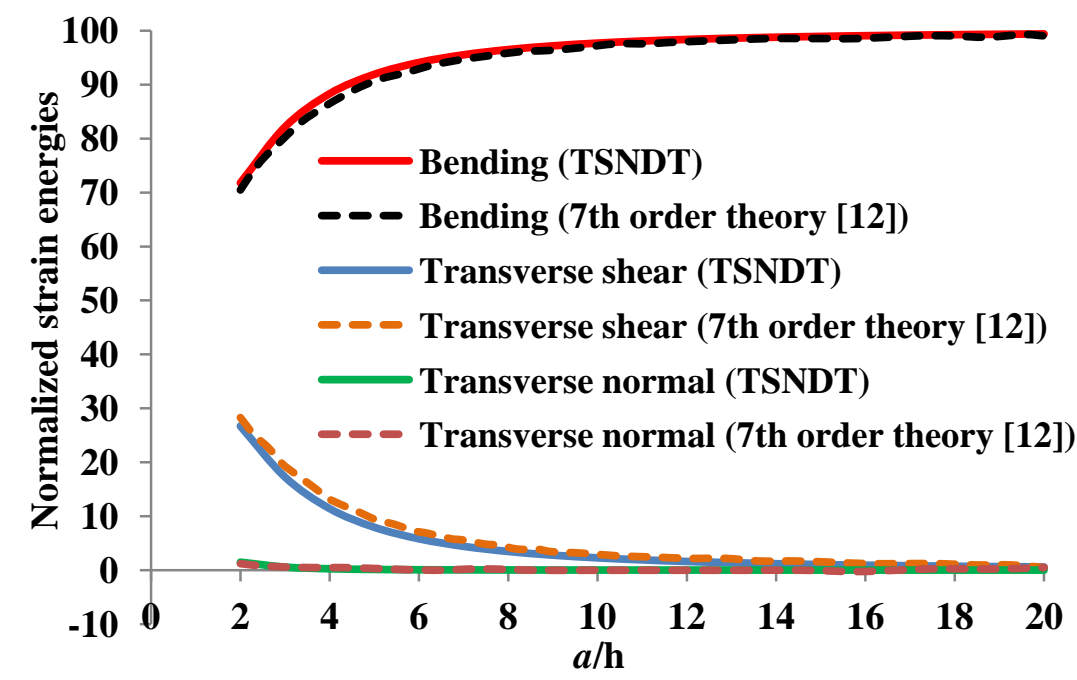

(a)

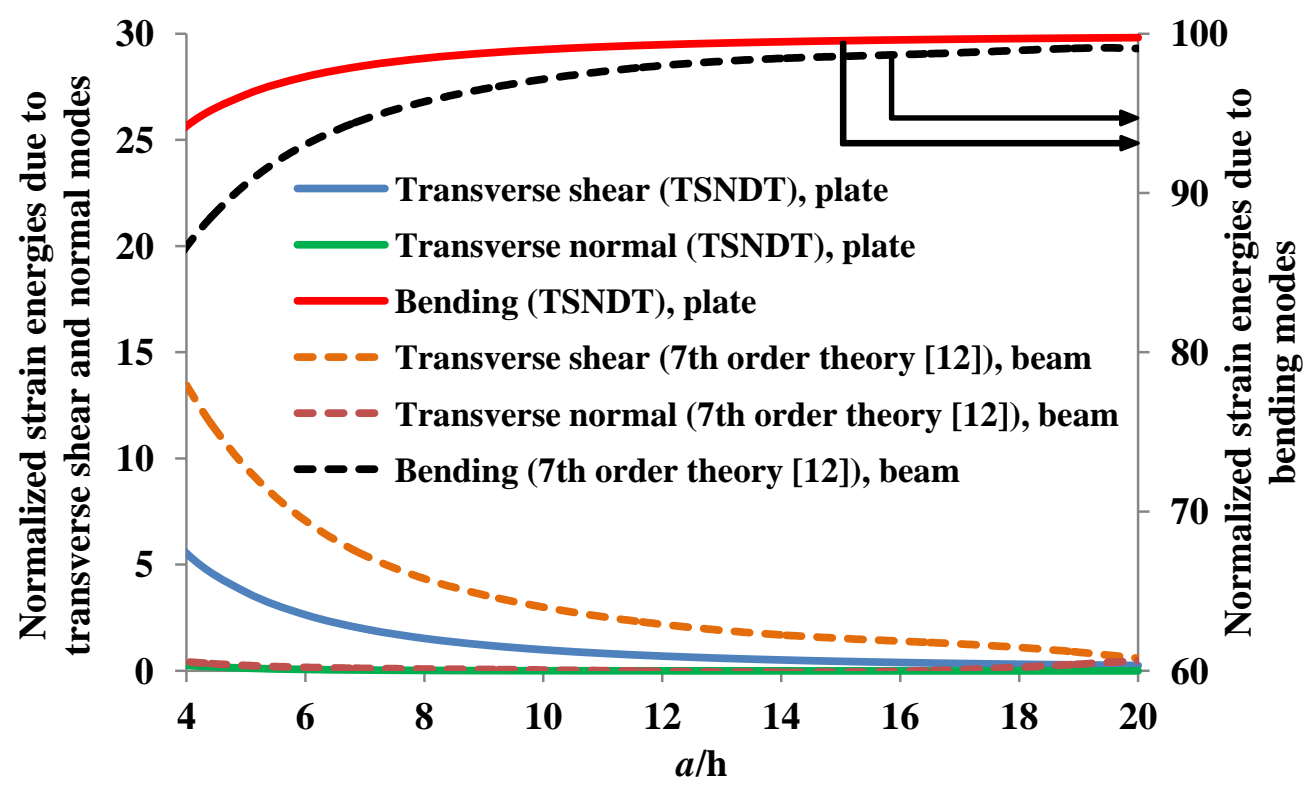

(b)

Fig. 6 Variation with the aspect ratio of the normalized values of the total strain energies of deformation due to bending, transverse shear and transverse normal deformations for an (a) orthotropic beam clamped at $\mathrm{x}=0$ and traction free at $\mathrm{x}=a$, and (b) orthotropic plate clamped at $\mathrm{x}=0$ and traction free on remaining edges, and subjected to equal and opposite uniform tangential tractions on the top and the bottom surfaces. Results from the $7^{\text {th }}$ order theory are plotted using the data digitized from [12]. 


\subsection{Combined tangential and normal uniform surface tractions on clamped $45^{\circ}$ composite plate}

We now analyze deformations of a clamped square plate with fibers oriented at $45^{\circ}$ to the $\mathrm{x}$ axis, having $\mathrm{h}=2.5 \mathrm{~cm}, a / \mathrm{h}=10$, values of material parameters listed in Data set 2 and only the top surface loaded by uniformly distributed normal $\left(\mathrm{q}_{\mathrm{z}}\right)$ and tangential $\left(\mathrm{q}_{\mathrm{x}}\right)$ tractions each of magnitude $10 \mathrm{MPa}$; the problem studied is schematically shown in inset of Fig. 7. In Fig. 7 we have depicted on the left and the right vertical axes, respectively, the $\mathrm{x}$ - and the $\mathrm{z}-$ displacements along the line $\mathrm{y}=b / 2$ on the mid-surface of the plate, i.e., $\mathrm{u}(\mathrm{x}, 0.5 b, 0)$ and $\mathrm{w}(\mathrm{x}, 0.5 b, 0)$, respectively. It is observed that the transverse normal displacement, $\mathrm{w}$, is one order of magnitude larger than the in-plane axial displacement, u. Also, displacements predicted by the TSNDT are in excellent agreement with the corresponding FE solutions of the 3-D LET equations with the maximum difference between two solutions being less than $0.12 \%$.

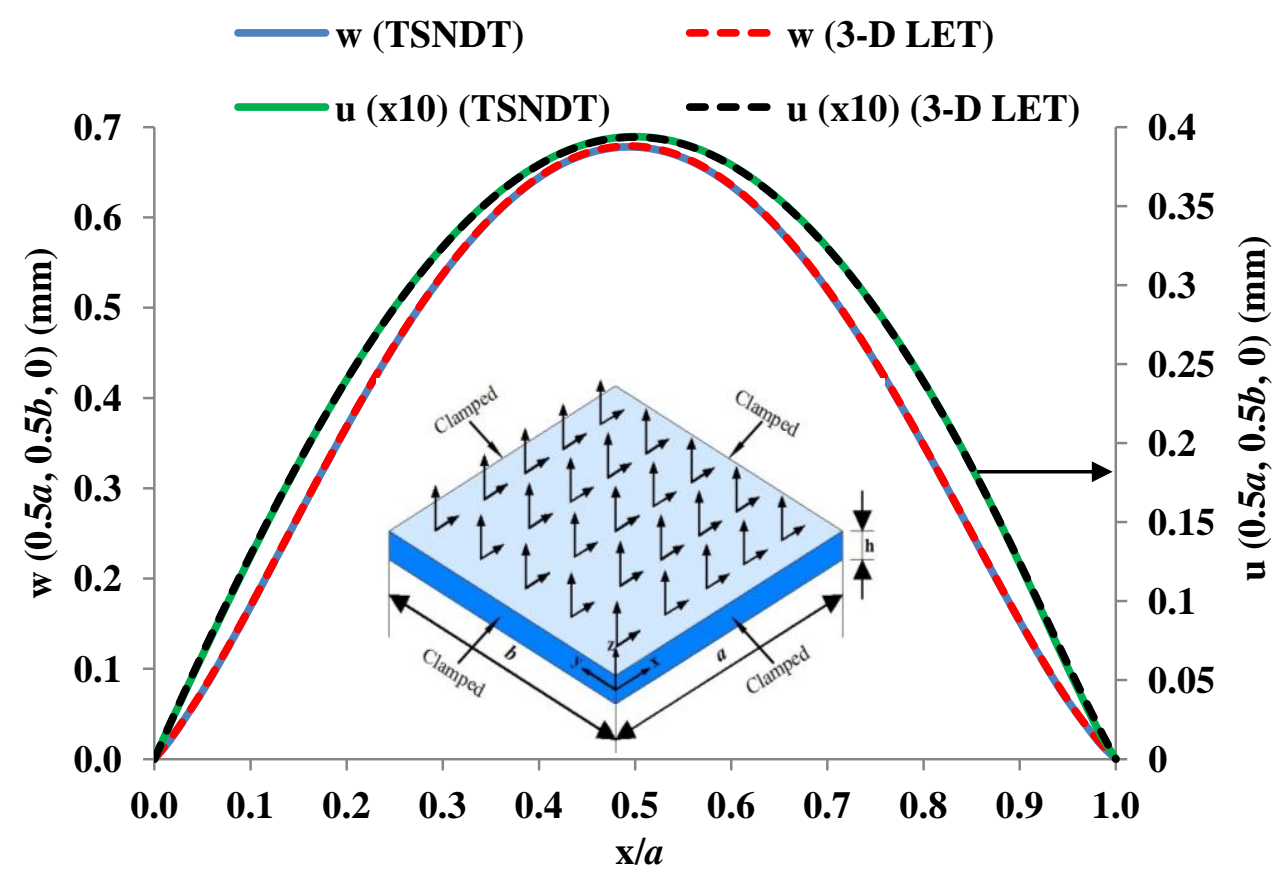

Fig. 7 For a clamped $45^{\circ}$ composite plate subjected to combined uniform normal and tangential tractions on its top surface, $\mathrm{x}$ - and $\mathrm{z}$ - components of displacement along the line $\mathrm{y}$ $=\mathrm{b} / 2$ on the mid-surface of the plate

In Figs. 8(a) and 8(b), we have displayed through-the-thickness distributions of the in-plane 
and the transverse stresses, respectively, at arbitrary points in the plate away from the edges. The results from the TSNDT at these points agree well with the corresponding FE solutions of the 3-D LET equations. It can be observed from the plots of Fig. 8(b) that the prescribed tangential traction boundary conditions are well satisfied on the top and the bottom surfaces of the plate. The errors in the computation of the tangential traction at the point $(0.167 a$, $0.167 b, 0.5)$ on the top surface are $0.23 \%$ and $0.98 \%$ when the stresses are computed from the constitutive relations and the SRS, respectively. The transverse normal stress predicted from the TSNDT deformations using the constitutive relation does not exhibit the "boundary layer" phenomenon near the major surfaces and the computed value at the centroid of the top surface differs from the applied traction by $10.6 \%$. However, this difference is reduced to $0.31 \%$ only and the "boundary layers" near the major surfaces are well captured when the stress is computed using the SRS.

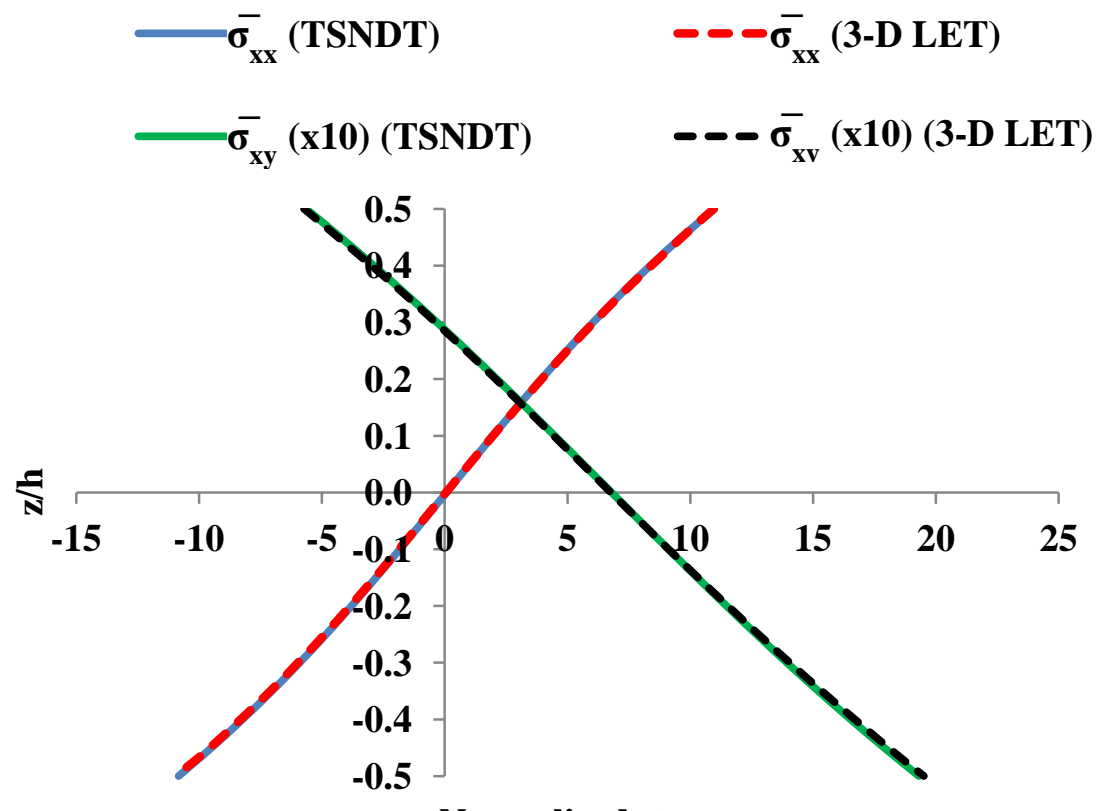

Normalized stress

(a) 

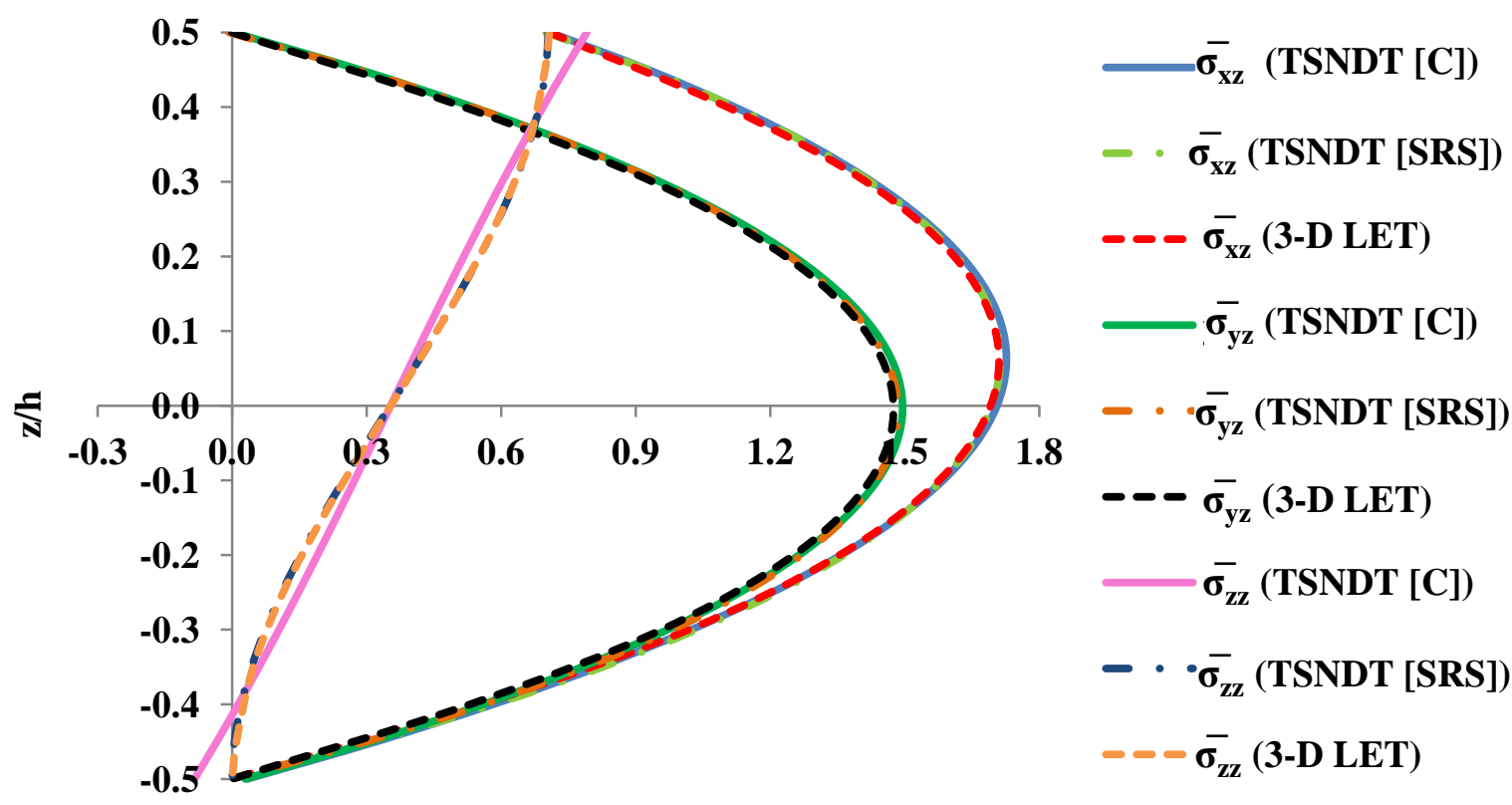

Normalized stress

(b)

Fig. 8 For a clamped $45^{\circ}$ composite plate subjected to uniform normal and tangential tractions on its top surface, through-the-thickness distribution of (a) $\bar{\sigma}_{\mathrm{xx}}(0.5 a, 0.5 b, \mathrm{z})$ and $\bar{\sigma}_{\mathrm{xy}}(0.25 a, 0.25 b, \mathrm{z})$, and (b) $\bar{\sigma}_{\mathrm{xz}}(0.167 a, 0.167 b, \mathrm{z}), \bar{\sigma}_{\mathrm{yz}}(0.167 a, 0.167 b, \mathrm{z})$, and $\bar{\sigma}_{\mathrm{zz}}(0.5 a, 0.5 b, \mathrm{z})$. The stresses are normalized by $\mathrm{q}_{0}=\sqrt{\mathrm{q}_{\mathrm{x}}^{2}+\mathrm{q}_{\mathrm{z}}^{2}}$.

In Figs. 9(a) and 9(b) we have portrayed through-the-thickness distributions of the transverse shear stress, $\sigma_{\mathrm{xz}}$, and the transverse normal stress at various sections near the edge $\mathrm{x}=0$. It is observed from Fig. 9(a) that the distribution of the transverse shear stress at the section $x=$ $0.05 a$ computed from the TSNDT using the constitutive relation differs at most by $5.6 \%$ from the 3-D FE solution and the tangential traction boundary conditions on the major surfaces are not well satisfied. When the SRS is employed to compute transverse stresses, this difference reduces to $1.5 \%$ and the tangential traction boundary conditions on the top and the bottom surfaces are accurately satisfied. However, the shear stress distribution at the section $\mathrm{x}=0.1 \mathrm{a}$ that is away from the edge $\mathrm{x}=0$ obtained using the TSNDT agrees well with that derived from the 3-D LET solution (3.3\% maximum difference at $(0.1 a, 0.5 b, 0))$ whether the stress is computed from the constitutive relations or the SRS. The results plotted in Fig. 9(b) reveal that $\sigma_{\mathrm{zz}}$ at two locations near the edge $\mathrm{x}=0$ computed from the constitutive relation do not 
satisfy the normal traction boundary conditions on the major surfaces and do not exhibit "boundary layer" effect as predicted by the 3-D LET. The transverse normal stresses at these locations, i.e., $\mathrm{x}=0.05 a$ and $0.1 a$, computed using the SRS accurately satisfy the normal traction boundary conditions on the top surface with $0.11 \%$ and $0.14 \%$ errors, respectively. However, they differ from the FE solutions of the corresponding 3-D LET equations by as much as $25.7 \%$ and $10.9 \%$, respectively, at $\mathrm{z}=-0.35 \mathrm{~h}$.

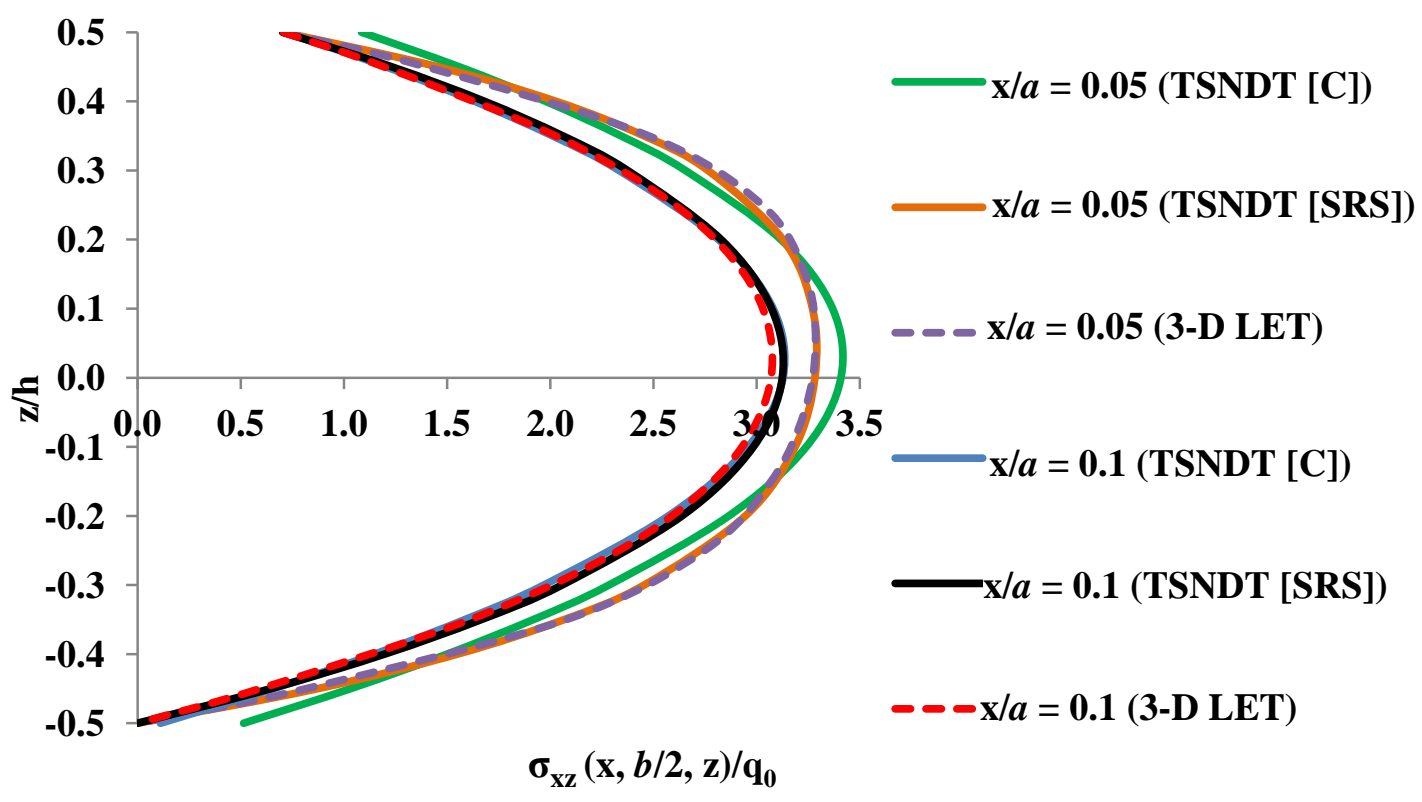

(a)

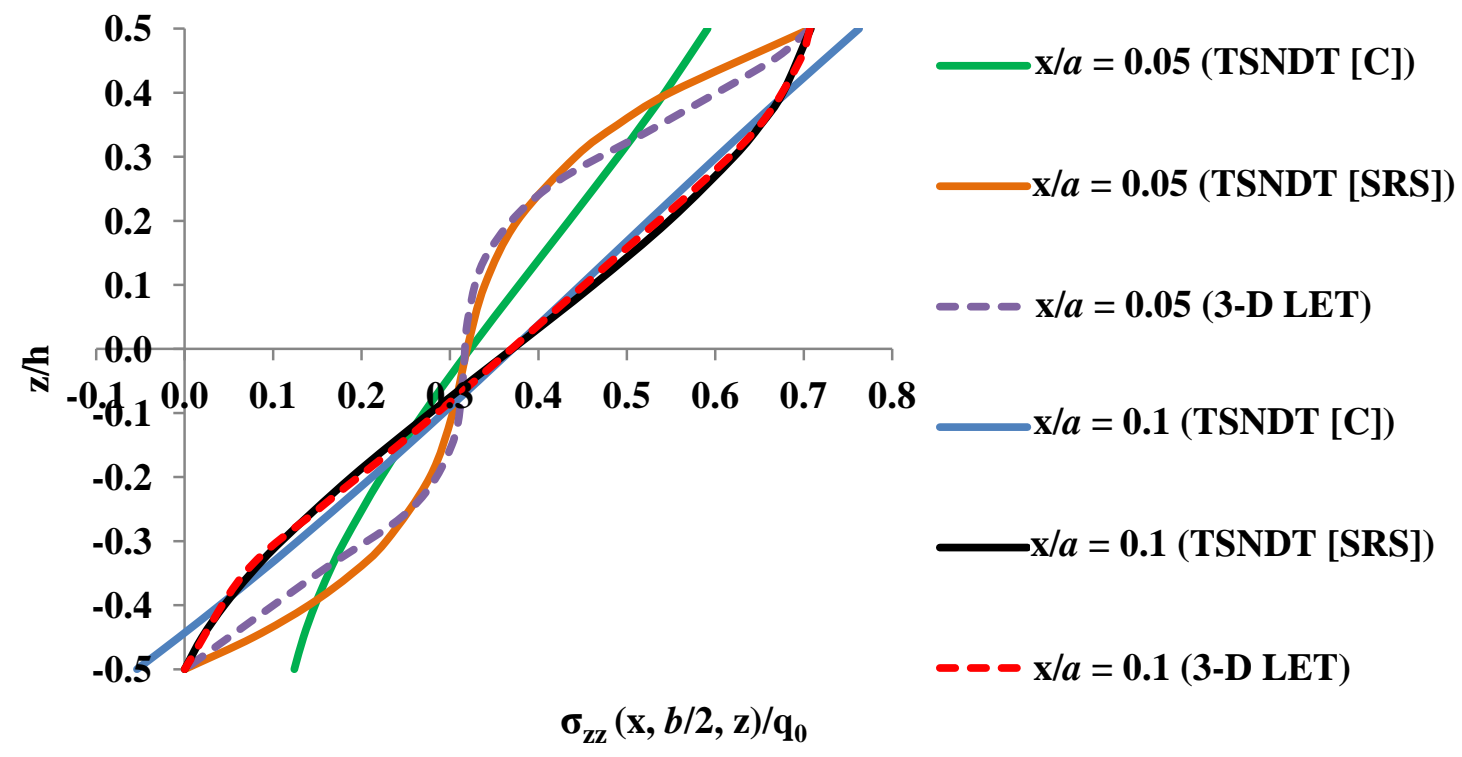

(b) 
Fig. 9 For a clamped $45^{\circ}$ composite plate subjected to uniform normal and tangential tractions on its top surface, through-the-thickness distribution of (a) $\sigma_{x z}(x, 0.5 b, z)$ and (b)

$$
\sigma_{\mathrm{zz}}(\mathrm{x}, 0.5 b, \mathrm{z}) \text { for } \mathrm{x} / a=0.05 \text { and } 0.1 \text {. }
$$

\subsection{Non-uniform pressure applied only on the top surface of clamped $45^{\circ}$ composite plate}

We study the problem defined in section 3.4 but with the following non-uniform pressure applied on plate's top surface with $a=22 \mathrm{~cm}$ and $a / \mathrm{h}=10$.

$$
\mathrm{P}(\mathrm{r})=\left\{\begin{array}{cc}
\mathrm{P}_{0}\left(-0.0005 \mathrm{r}^{4}+0.01 \mathrm{r}^{3}-0.0586 \mathrm{r}^{2}-0.001 \mathrm{r}+1\right), & \mathrm{r}<10.6 \mathrm{~cm} \\
0, & \mathrm{r} \geq 10.6 \mathrm{~cm}
\end{array}\right.
$$

Here $\mathrm{r}$ is the distance in $\mathrm{cm}$ from the centroid of the top surface, and $\mathrm{P}_{0}=10 \mathrm{MPa}$. We note that the polynomial function given by Eq. (14) represents a typical distribution of pressure on the plate surface due to an underwater explosion, e.g., see [51]. This pressure profile is schematically depicted in the inset of Fig. 10.

The two distributions of the displacement, $\mathrm{w}(\mathrm{x}, b / 2,0)$, of plate's mid-surface along the line $\mathrm{y}=b / 2$ found by using the TSNDT and by solving the 3-D LET equations plotted in Fig. 10 differ from each other by less than 0.02\%. In Figs. 11(a) and 11(b), we have displayed through-the-thickness distributions of the in-plane and the transverse stresses, respectively, at arbitrarily chosen points away from the plate edges. For this problem also, the through-thethickness stress distributions computed using the SRS and the FEM applied to solve the 3-D LET equations agree well with each other and the transverse normal stress shows the "boundary layer" phenomenon. The difference in the normal traction at the centroid of the top surface of the plate computed from the constitutive relations and the SRS equals $-11.6 \%$ and $-0.77 \%$, respectively. 


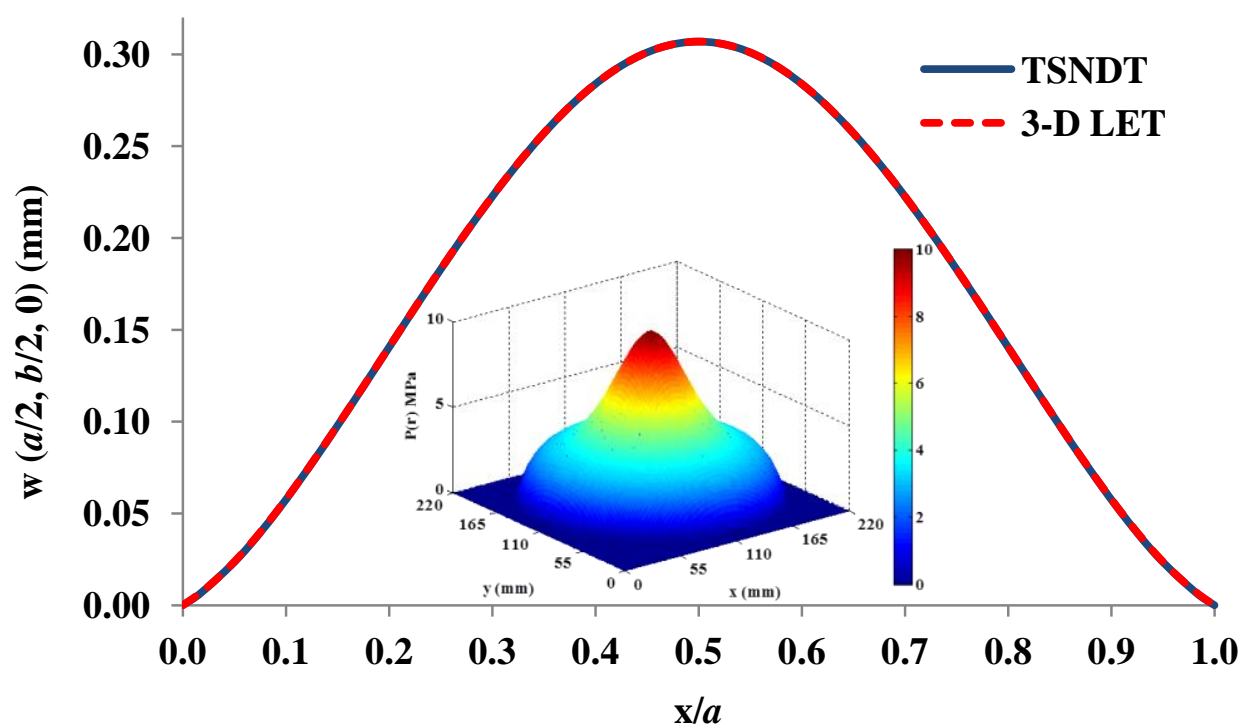

Fig. 10 For a clamped $45^{\circ}$ composite plate subjected to pressure given by Eq. (14) on its top surface, $\mathrm{z}$ - component of displacement along the line $\mathrm{y}=\mathrm{b} / 2$ on the mid-surface of the plate

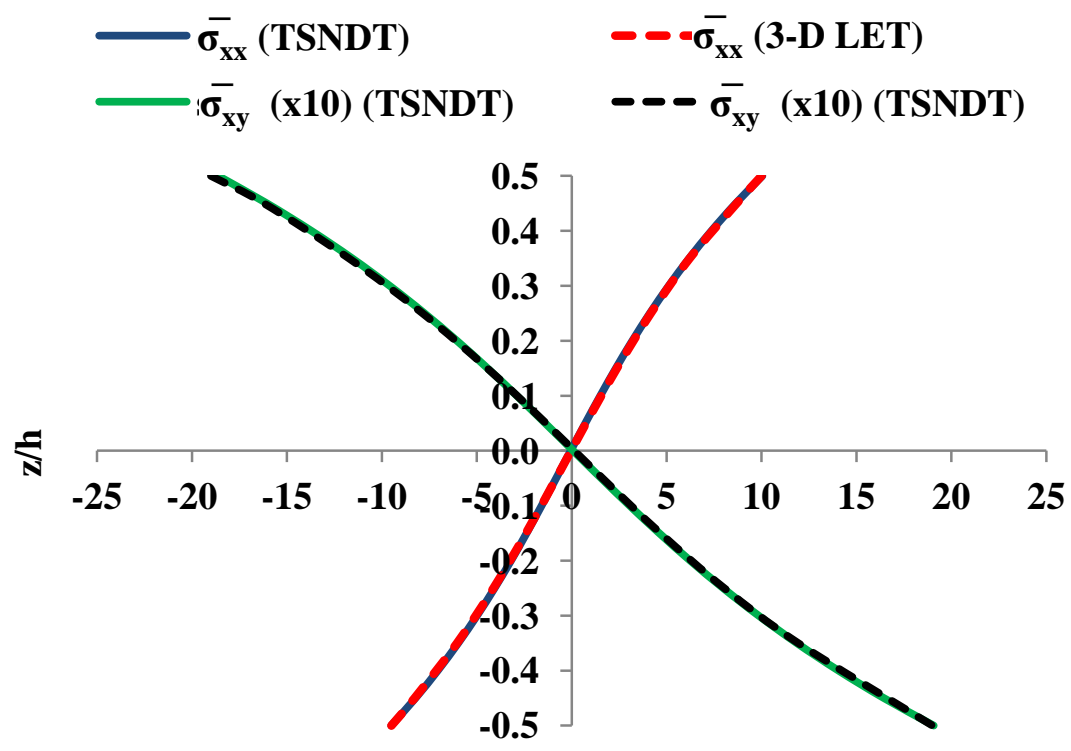

Normalized stress

(a) 


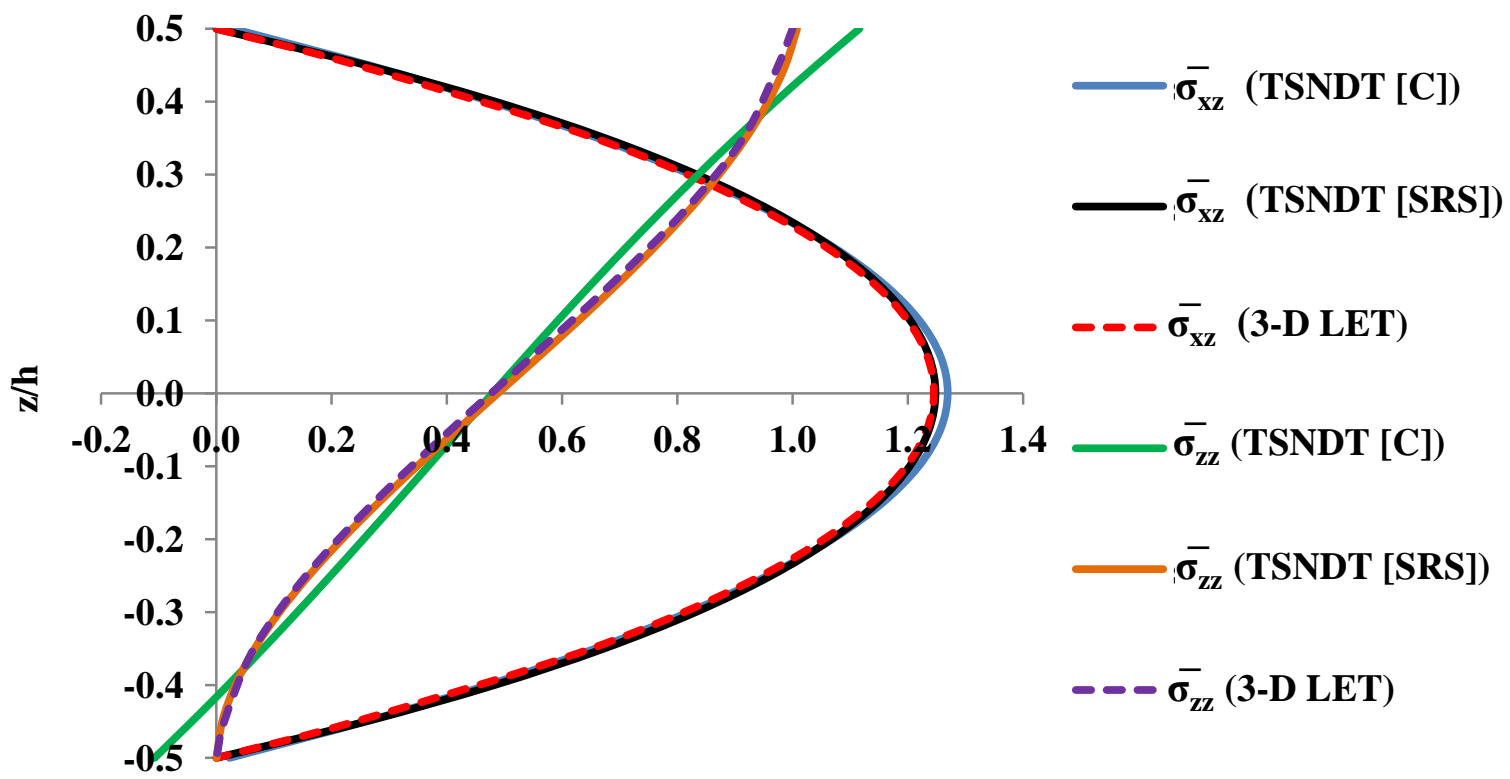

Normalized stress

(b)

Fig. 11 For a clamped $45^{\circ}$ composite plate subjected to pressure given by Eq. (14) on its top surface, through-the-thickness distributions of (a) $\bar{\sigma}_{\mathrm{xx}}(0.5 a, 0.5 b, \mathrm{z})$ and $\bar{\sigma}_{\mathrm{xy}}(0.25 a, 0.25 b, \mathrm{z})$, and (b) $\bar{\sigma}_{\mathrm{xz}}(0.25 a, 0.5 b, \mathrm{z})$ and $\bar{\sigma}_{\mathrm{zz}}(0.5 a, 0.5 b, \mathrm{z})$. The stresses are normalized by $\mathrm{P}_{0}$.

In Fig. 12 we have displayed the applied normal traction and the distribution of the transverse normal stress on the top surface along the $y=b / 2$ computed from the TSNDT and the 3-D LET. The transverse normal stress computed from the TSNDT using the constitutive relation and the SRS satisfy the normal traction boundary condition on the top surface with maximum errors of $11.86 \%$ and $1.63 \%$, respectively, in the region between $\mathrm{x}=0.1 a$ and $\mathrm{x}=0.9 a$. At $(0.056 a, 0.5 b, 0.5 \mathrm{~h})$, these errors increase to $38 \%$ and $5 \%$, and become even larger with a decrease in the distance from the edge $\mathrm{x}=0$. The transverse normal stress at points located at a distance smaller than $10 \%$ edge-length computed using the SRS is found to be more accurate than that obtained from the 3-D LET and the FEM. We note that in the displacement based FE formulation, the stresses at nodes are not accurately computed. Hence, we obtain the value of $\sigma_{\mathrm{zz}}$ at a clamped edge using the least square fit to that evaluated at integration points in the element adjacent to the edge. At the risk of repetition, we mention that for the 8node serendipity element, Barlow proved that stresses at the $2 \times 2$ quadrature points have the 
least error [52]. At the edge $\mathrm{x}=0, \sigma_{\mathrm{zz}}$ computed from the 3-D LET and the TSNDT using the constitutive relation as well as the SRS does not equal zero even though the applied normal traction there is zero. We note that the 3-D LET solution has been found by using the $100 \times 100 \times 10 \mathrm{FE}$ mesh of uniform 8-node brick elements. Stresses are first computed at the $2 \times 2 \times 2$ quadrature points in each element, first extrapolated to the top surface, and then from there to the entire surface. We have not experimented with different FE meshes to see if the mesh refinement especially near the clamped edges will better satisfy the traction boundary conditions at points close to clamped edges. In Fig. 13 we have depicted throughthe-thickness distribution of $\sigma_{\mathrm{xz}}$ at the section $\mathrm{x}=0.05 \mathrm{a}$. The stress obtained from the constitutive relation is not accurate and does not satisfy the tangential traction boundary conditions on the major surfaces. The difference in maximum value of $\sigma_{\mathrm{xz}}$ at $\mathrm{z}=0$ thus obtained and that given by the 3 -D LET is $5.5 \%$. This difference reduces to $2.4 \%$ when $\sigma_{\mathrm{xz}}$ is computed using the SRS.

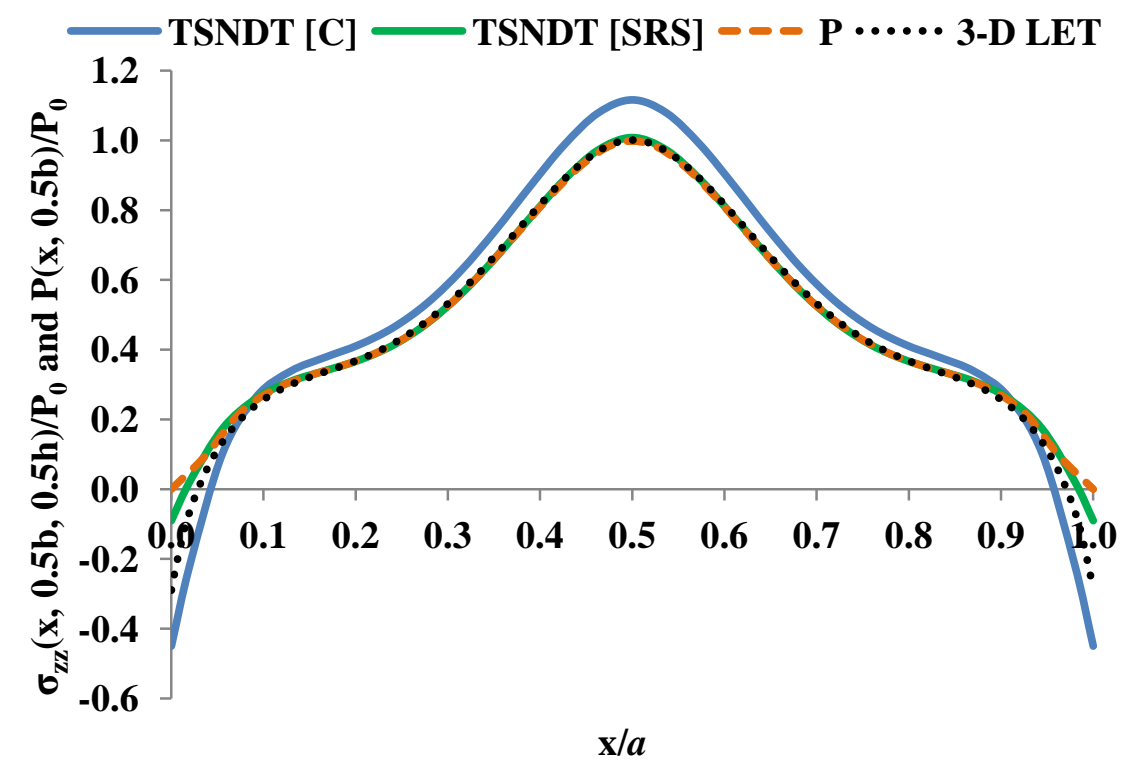

Fig. 12 For a clamped $45^{\circ}$ composite plate subjected to pressure given by Eq. (14) on its top surface, $\sigma_{\mathrm{zz}}(\mathrm{x}, 0.5 \mathrm{~b}, 0.5 \mathrm{~h})$ along the line $\mathrm{y}=\mathrm{b} / 2$ on the top surface of the plate 


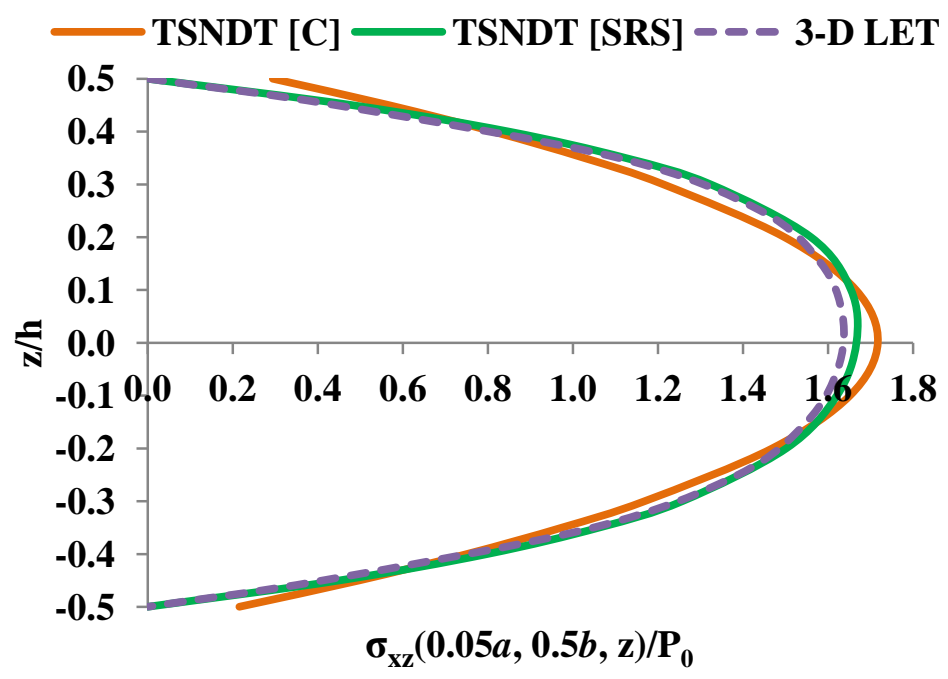

Fig. 13 For a clamped $45^{\circ}$ composite plate subjected to pressure given by Eq. (14) on its top surface, through-the-thickness distribution of $\sigma_{\mathrm{xz}}(0.05 a, 0.5 b, \mathrm{z})$

\subsection{Angle-ply $45^{\circ} \% 0^{\circ} 45^{\circ}$ laminated plate subjected to uniform normal traction on the top surface}

We study deformations of a clamped square angle-ply $45^{\circ} / 0^{\circ} / 45^{\circ}$ laminate with $\mathrm{h}=3 \mathrm{~cm}$ (each layer is $1 \mathrm{~cm}$ thick), $a / \mathrm{h}=10$, having values of material constants listed in Data set 2 and subjected to a uniform normal tensile traction, $\mathrm{q}_{0}=10 \mathrm{MPa}$, only on its top surface. In Fig. 14(a),(b) we have displayed along the transverse normal passing through the plate centroid through-the-thickness distributions of the in-plane axial stresses obtained from the constitutive relations and the transverse normal stress computed using the SRS, respectively. Values of stresses $\sigma_{\mathrm{xx}}$ and $\sigma_{\mathrm{yy}}$ computed from the constitutive relations differ from those given by the solution of the 3-D LET equations by a maximum of $6.95 \%$ and $6.25 \%$ at the interface of the top and the middle layer. In Fig. 14(c) we have depicted through-thethickness variations of the transverse shear stresses, $\sigma_{\mathrm{xz}}$ and $\sigma_{\mathrm{yz}}$, computed from the TSNDT using the SRS and those obtained from the 3-D FEM along the transverse normal passing through points $(0.1 a, 0.5 b, 0)$ and $(0.5 a, 0.1 b, 0)$, respectively. The two sets of results agree well with $2.16 \%$ and $5.67 \%$ maximum difference in $\sigma_{\mathrm{xz}}$ and $\sigma_{\mathrm{yz}}$, respectively, at $\mathrm{z}=0$ on the respective sections. Since transverse stresses computed from the constitutive relations for a 
laminated plate are not accurate and continuous across the interface between two adjacent layers, they are not plotted in Fig. 14. We note that the 3-D FE solution is obtained with a uniform $60 \times 60 \times 6$ mesh of 8-node brick elements in each layer (thus a total 234,423 DoFs).

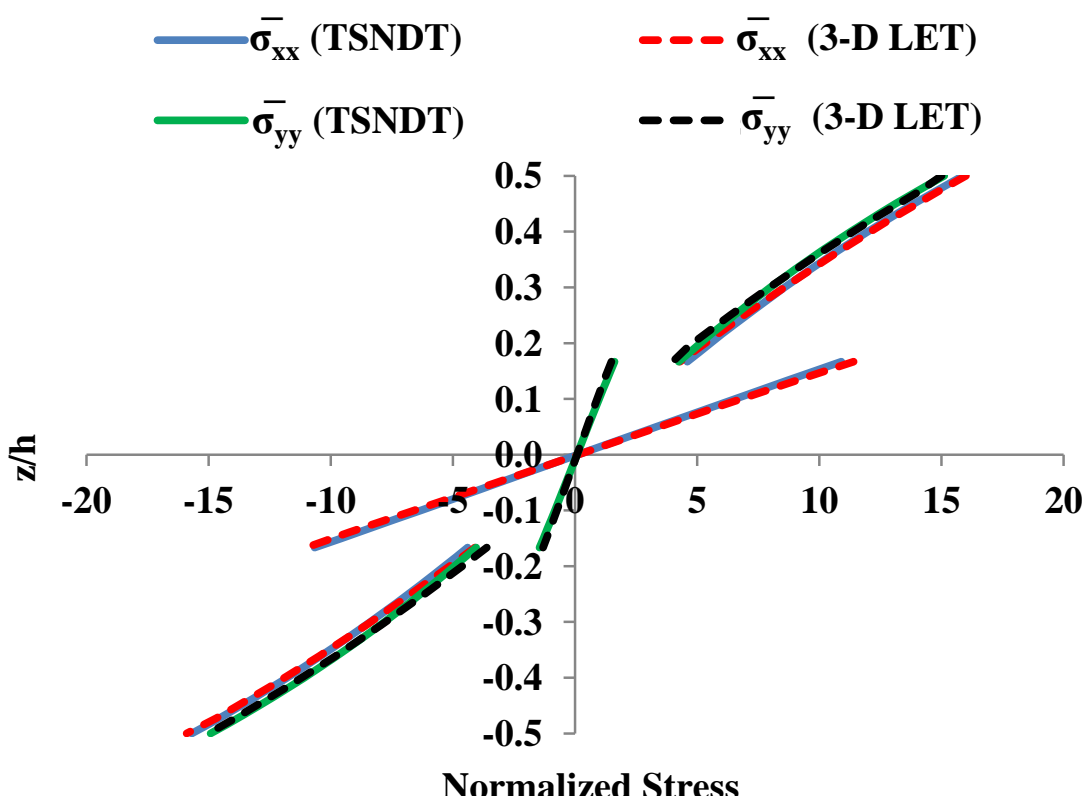

(a)

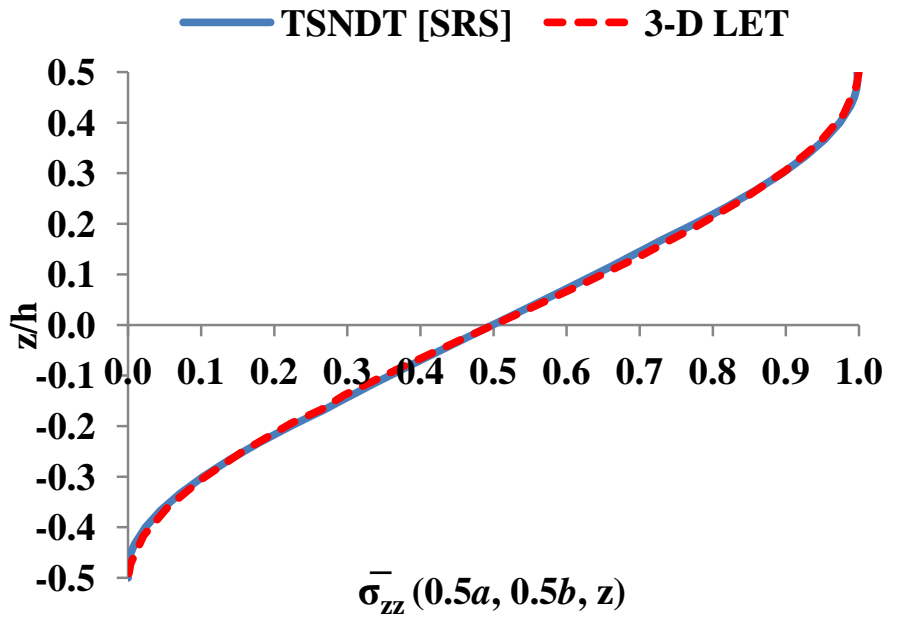

(b) 


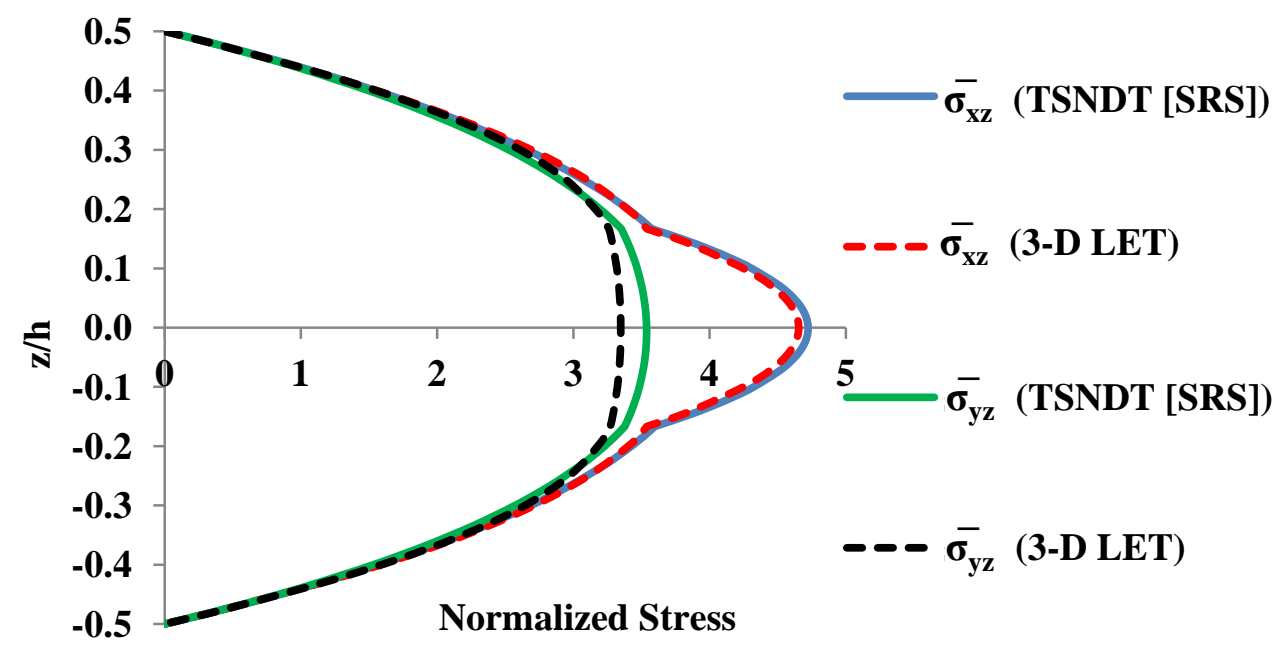

(c)

Fig. 14 For a clamped $45^{\circ} / 0^{\circ} / 45^{\circ}$ composite plate subjected to uniform normal tensile traction on its top surface, through-the-thickness distribution of (a) $\bar{\sigma}_{\mathrm{xx}}(0.5 a, 0.5 b, \mathrm{z})$ and $\bar{\sigma}_{\mathrm{yy}}(0.5 a, 0.5 b, \mathrm{z}),(\mathrm{b}) \bar{\sigma}_{\mathrm{zz}}(0.5 a, 0.5 b, \mathrm{z})$, and (c) $\bar{\sigma}_{\mathrm{xz}}(0.1 a, 0.5 b, \mathrm{z})$ and $\bar{\sigma}_{\mathrm{yz}}(0.5 a, 0.1 b, \mathrm{z})$. The stresses are normalized by $\mathrm{q}_{0}$.

\subsection{Cross-ply $0^{\circ} / 90^{\circ} / 0^{\circ}$ laminated plate subjected to sinusoidal tensile normal traction on the top surface}

We study deformations of a thick symmetric cross-ply $0 \% 190^{\circ} / 0^{\circ}$ laminated square plate having $a / h=5$ with equally thick layers, values of material parameters listed in Data set 1 , with the traction free bottom surface and subjected to a sinusoidal distributed tensile normal traction given by Eq. (15) on the top surface.

$\mathrm{q}(\mathrm{x}, \mathrm{y})=\mathrm{q}_{0} \sin (\pi \mathrm{x} / a) \sin (\pi \mathrm{y} / b)$

The edges $\mathrm{y}=0$ and $b$ are simply supported and the edges $\mathrm{x}=0$ and $a$ are either clamped or simply supported (BCs A or B in Table 1). In Fig. 15(a),(b) we have displayed through-thethickness distributions of the transverse shear stress, $\sigma_{\mathrm{yz}}$, near the edge $\mathrm{x}=0$ and along the line $\mathrm{y}=b / 2$ on the mid-surface of the laminate obtained with the TSNDT using the SRS and the corresponding solution given by Vel and Batra [53] who obtained an analytical solution of the problem based on the 3-D LET using the generalized Eshelby-Stroh formalism. The plots in Fig. 15 are labeled as AA and BB, respectively, for BCs A and B prescribed on edges 
$\mathrm{x}=0$ and $a$. It is evident from the plots of Fig. 15(a) that the SRS captures the presence of "boundary layers" near the major surfaces of the laminate with clamped opposite edges. This "boundary layer" is absent when all edges of the laminate are simply supported. The results plotted in Fig. 15(b) show that the TSNDT accurately predicts the distribution of the transverse shear stress along the span of the laminate. Here also the stress at an edge is computed by using the least square fit to its values at integration points in the element adjacent to the edge. The maximum difference between the values of $\sigma_{y z}$ obtained from the TSNDT and the 3-D analytical solution is $7.2 \%$ and occurs at the clamped edges $\mathrm{x}=0$ and $a$.

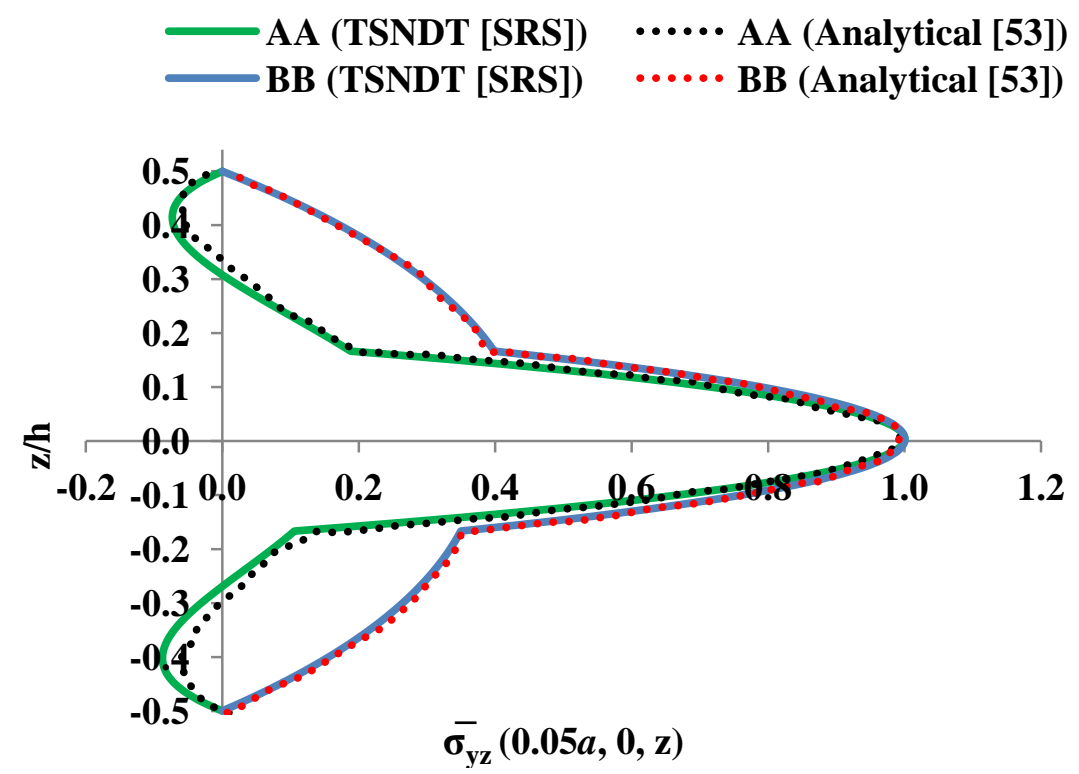

(a)

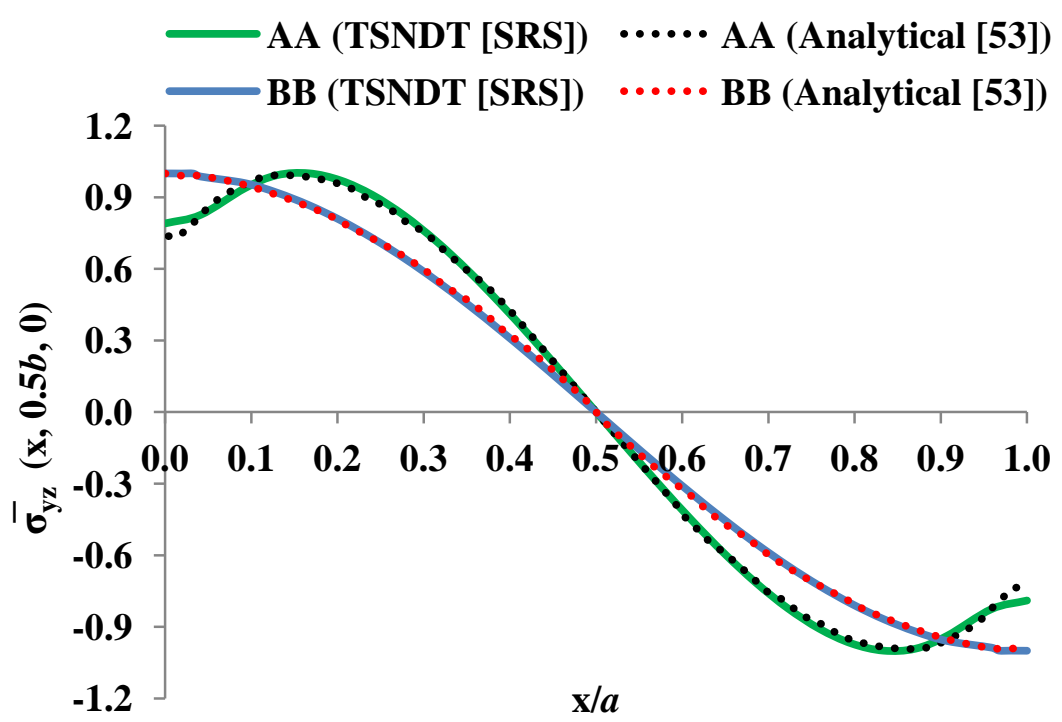


(b)

Fig. 15 For a $0^{\circ} / 90^{\circ} / 0^{\circ}$ composite plate subjected to the loading of Eq. (15) on its top surface, distributions of the normalized transverse shear stress, $\bar{\sigma}_{y z}$ (a) through the thickness along the transverse normal passing through $(0.05 a, 0,0)$, and (b) along the line $\mathrm{y}=b / 2$ on the midsurface of the laminate for two BCs. The stress in each plot is normalized by its maximum value. Analytical solutions are plotted using the data digitized from [53].

In Fig. 16 we have displayed the effect of BCs on through-the-thickness variation of the transverse normal stress near the edge $x=0$ of the laminate. Here, one more case is considered in which the edges $\mathrm{y}=0$ and $b$ are simply supported and the other two edges are traction free (indicated by the plot marked as FF in Fig. 16). The transverse normal stress also exhibits the "boundary layer" effect for the BCs other than all edges simply supported. The results from the TSNDT match well with the analytical solution when edges $\mathrm{x}=0$ and $a$ are simply supported or traction free. When these edges are clamped, the TSNDT does not accurately predict the stress distribution and the maximum difference between the two solutions is $41 \%$ at $\mathrm{z}=0.27 \mathrm{~h}$. The percentage errors between the computed traction on the top surface at the point $(0.075 a, 0.5 b, 0.5 \mathrm{~h})$ using the SRS and the applied traction are $0.56 \%$, $0.28 \%$ and $2.48 \%$ for BCs AA, BB and FF, respectively.

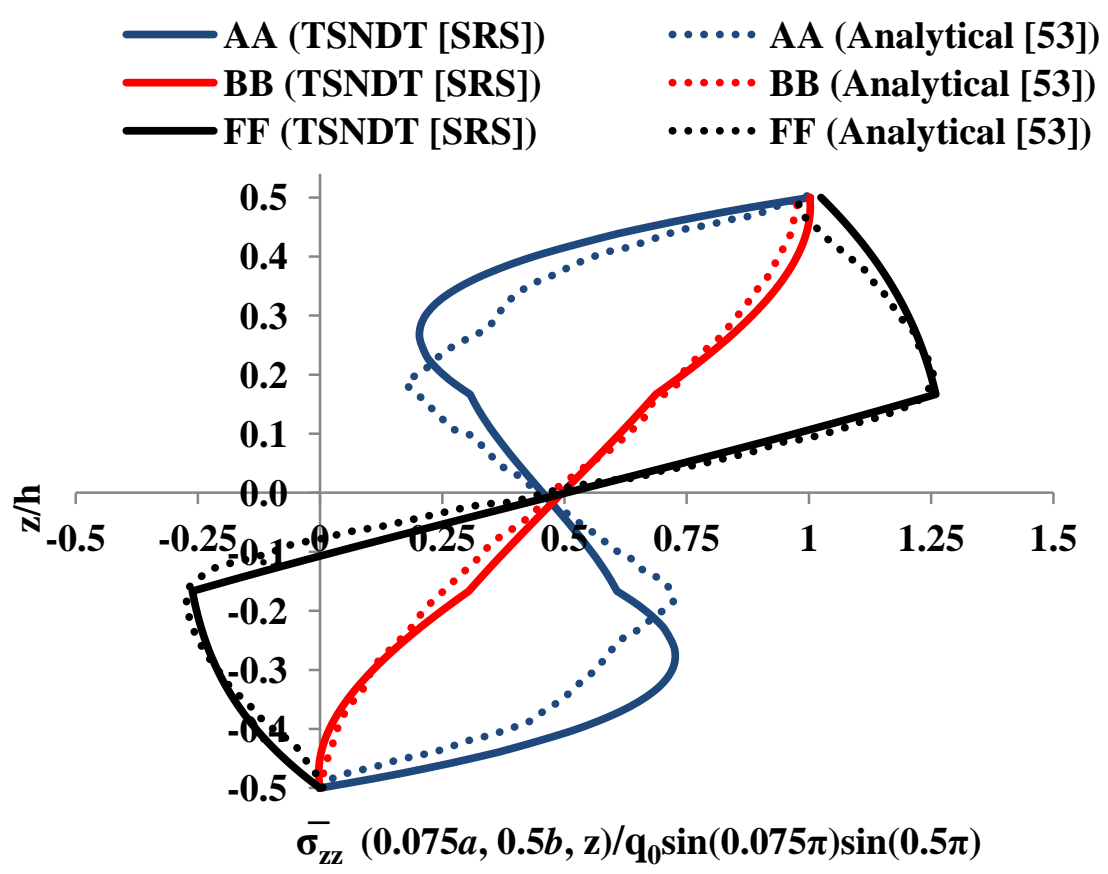


Fig. 16 For a $0^{\circ} / 90^{\circ} / 0^{\circ}$ composite plate subjected to the loading of Eq. (15) on its top surface, through-the-thickness distribution of normalized $\sigma_{\mathrm{zz}}(0.075 a, 0.5 b, \mathrm{z})$ for three BCs. Analytical solutions are plotted using the data digitized from [53].

\subsection{Cross-ply $0^{\circ} / 90^{\circ}$ laminated plate subjected to sinusoidally distributed tensile normal traction on the top surface}

We now study deformations of a thick anti-symmetric cross-ply $0^{\circ} / 90^{\circ}$ laminated square plate with $a / \mathrm{h}=5$ (both layers are equally thick), material properties given by Data set 1 and subjected to the sinusoidally distributed tensile normal traction given by Eq. (15) only on its top surface. The edges y $=0$ and $b$ are simply supported and four different BCs denoted by A, $\mathrm{B}, \mathrm{C}$ and D (see Table 1) are specified on the edges $\mathrm{x}=0$ and $a$. In Fig. 17 we have plotted through-the-thickness distributions of the transverse shear stress, $\sigma_{\mathrm{yz}}$, near the edge $\mathrm{x}=0$ obtained from the TSNDT using the SRS and that reported by Vel and Batra [53].

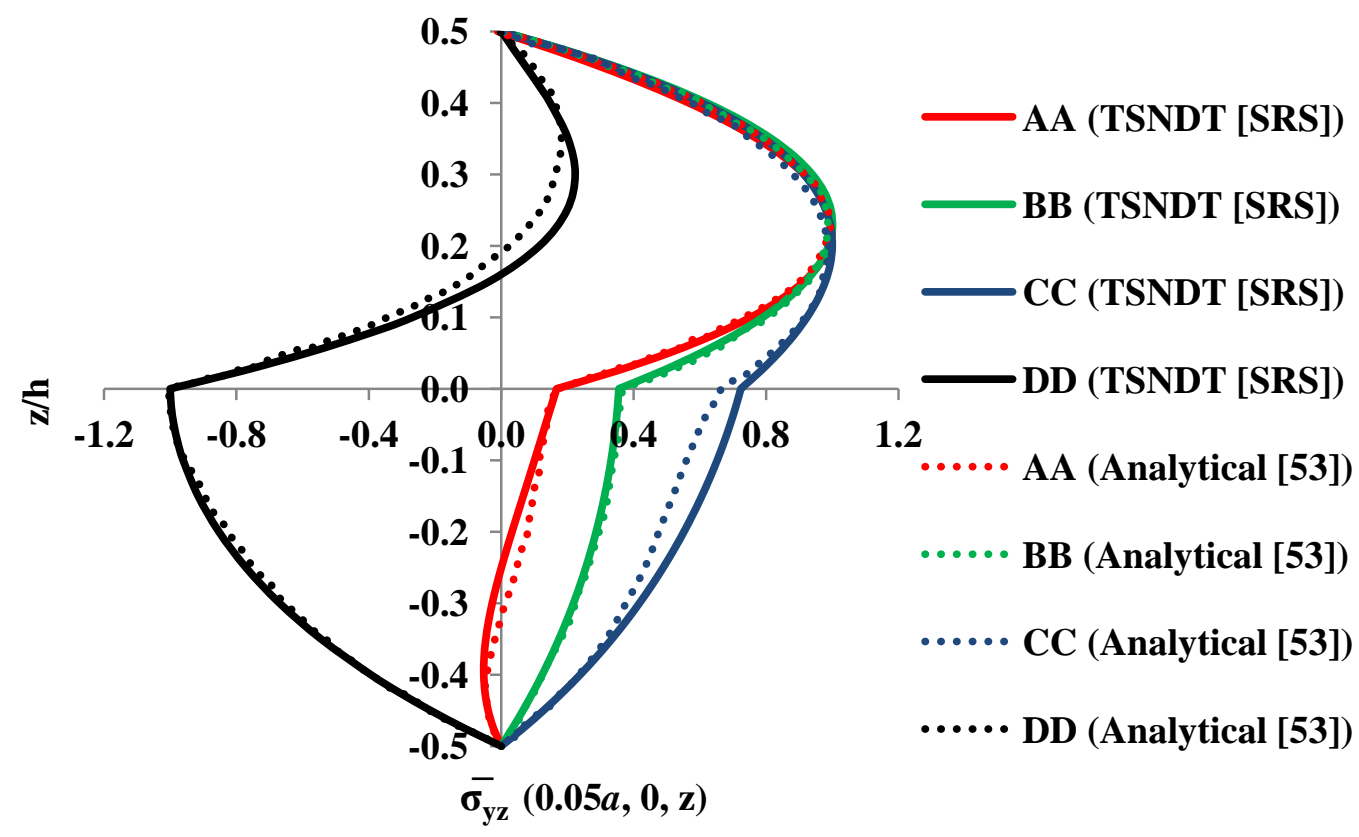

Fig. 17 For a $0^{\circ} / 90^{\circ}$ composite plate subjected to the loading of Eq. (15) on its top surface, through-the-thickness distribution of $\bar{\sigma}_{\mathrm{yz}}(0.05 a, 0.5 b, \mathrm{z})$ for four BCs. The stress is normalized by its maximum value. Analytical solutions are plotted using the data digitized from [53]. 
It is evident that the through-the-thickness distribution of the shear stress at a point near the boundary depends upon the type of the BC. When the edges $\mathrm{x}=0$ and $a$ are prescribed with A or D BCs, the "boundary layer" phenomenon is observed near the bottom surface of the plate. The TSNDT coupled with the SRS accurately predicts the distribution when the edges $\mathrm{x}=0$ and $a$ are clamped (A) or simply supported (B). The maximum difference between the TSNDT and the analytical solutions equals $7.6 \%, 18.18 \%$ and $40.27 \%$ for AA, CC and DD BCs and it occurs at $\mathrm{z}=-0.25 \mathrm{~h},-0.1 \mathrm{~h}$ and $0.27 \mathrm{~h}$, respectively. This difference can be reduced either by using a layerwise theory or by employing a higher order shear and normal deformable plate theory or by using the Hellinger-Prange-Reissner variational principle to drive the plate theory. We note that for a simply supported $0^{\circ} / 90^{\circ} / 0^{\circ}$ laminated beam loaded by sinusoidal normal traction, Batra and Xiao [54] found accurate values of the transverse shear stresses at the edge by using a layerwise TSNDT.

\section{Conclusions}

A third order shear and normal deformable plate theory (TSNDT) has been used for analyzing static infinitesimal deformations of composite laminated plates made of linear elastic orthotropic materials. The finite element method (FEM) is used to obtain numerical solutions for seven example problems using the TSNDT and considering the laminate as a single layer. No shear correction factor is used. For monolithic plates, stresses are obtained directly from the constitutive relations. However, for multi-layered plates transverse shear and normal stresses are computed using a stress recovery scheme (SRS).

For monolithic plates, in-plane stresses and transverse shear stresses at points situated at a distance greater than $10 \%$ edge-length are accurately computed directly from the constitutive relations without using the SRS. However, near an edge they are not accurate and differ at some points by about $45 \%$ from the corresponding 3-D linear elasticity theory (LET) solutions for some cases of applied tractions and boundary conditions at the edges. Also the transverse normal stress computed on the major surfaces differs from the applied normal traction by about $10-20 \%$, and the "boundary layer" phenomenon near these surfaces is not captured. However, when the SRS is employed the transverse normal stresses satisfy the traction boundary conditions on the major surfaces with less than $3 \%$ error, exhibit the 
"boundary layer" effect as predicted by the 3-D LET, and through-the-thickness stress distributions in the vicinity of an edge are accurately computed. For problems involving tangential tractions on the major surfaces of the plate, results from the TSNDT differ by less than $3 \%$ from those predicted by a $6^{\text {th }}$ order shear and normal deformable plate theory and the 3-D LET for span/thickness $\geq 5$. For a thick plate with span/thickness $=2$, this difference increases to about $11 \%$.

For laminated plates, through-the-thickness distribution of transverse stresses near an edge is found to depend on the type of boundary condition (BC) specified on that edge. For a plate with two opposite edges simply supported and the other two edges either clamped or traction free, transverse stresses in the vicinity of the clamped or the free edge exhibit "boundary layer" effect near the major surfaces which is found to be absent when all edges of the plate are simply supported. For a moderately thick laminated plate (span/thickness $=10$ ), the SRS accurately predicts the stress distributions near an edge. For a thick laminated plate (span/thickness $=5)$, the SRS accurately predicts the stress distributions in the vicinity of an edge $(($ distance from the edge $) /$ span $=0.05)$ when all edges are simply supported. However, for some other BCs up to $40 \%$ maximum difference between the results from the TSNDT coupled with the SRS and the 3-D LET occur near an edge.

For an aspect ratio of 10 (5) the strain energy due to transverse shear deformations equals $3 \%(9.3 \%)$ of the total strain energy for a cantilever beam but only $1 \%(3.7 \%)$ for a cantilever plate with equal and opposite tangential tractions applied on the major surfaces.

The present work establishes the applicability of the TSNDT coupled with the SRS in predicting accurate responses of the composite laminated plates for a wide range of problems without using a shear correction factor and with an advantage of requiring considerably less degrees of freedom than those needed for solving the problem with the FEM and using the 3D LET. However, at points near clamped and free edges of a laminate, one needs to check the accuracy of the computed stresses by studying the corresponding 3-D problem especially for failure/damage initiation and propagation.

\section{Acknowledgements:}

This work was partially supported by the Office of Naval Research grant N00014-11-1-0594 to Virginia Polytechnic Institute and State University with Dr. Y. D. S. Rajapakse as the 
Program Manager. Views expressed in the paper are those of the authors and neither of the funding agency nor of authors' institutions. 


\section{References}

1. Kirchhoff, G. Über das Gleichgewicht und die Bewegung einer elastischer Scheibe. Zeitschr. reine und angewandte Math, 1850. 40(1): p. 51-88.

2. Cosserat, E. and F. Cosserat, Sur la statique de la ligne deformable. Comptes Rendus Chimie, 1907. 145: p. 1409-1412.

3. Ericksen, J.L., Plane infinitesimal waves in homogeneous elastic plates. Journal of Elasticity, 1973. 3(3): p. 161-167.

4. Koiter, W.T and J.G. Simmonds, Foundations of shell theory. In: Proc. IUTAM Congress, Moscow, 1972. pp. 150-176

5. Naghdi, P.M., The theory of shells and plates. Springer Berlin Heidelberg, 1973. p. 425-640.

6. Antman, S.S., The theory of rods. In: C. Truesdell (ed.), Handbuch der Physik, 1972. Vol. VI a/2. Springer, Berlin (1972).

7. Leissa, A.W., Recent research in plate vibrations, Part I: Classical theory; Part II: Complicating effects, 1981-85. The Shock and Vibration Digest, 1987, 19(2): p. 11-18; 1987, 19(3): p. 10-24.

8. Carrera, E., Theories and finite elements for multilayered plates and shells: a unified compact formulation with numerical assessment and benchmarking. Archives of Computational Methods in Engineering, 2003. 10(3): p. 216-296.

9. Mindlin, R.D. and M.A. Medick. Extensional vibrations of elastic plates. Journal of applied Mechanics, 1959. 26(4): p. 561-569.

10. Teresi, L. and A. Tiero, On variational approaches to plate models. Meccanica, 1997. 32(2): p. 143-156.

11. Vidoli, S. and R.C. Batra, Derivation of plate and rod equations for a piezoelectric body from a mixed three-dimensional variational principle. Journal of Elasticity, 2000. 59(1-3): p. 23-50.

12. Batra, R.C. and S. Vidoli, Higher-order piezoelectric plate theory derived from a threedimensional variational principle. AIAA Journal, 2002. 40(1): p. 91-104.

13. Batra, R.C., Vidoli, S. and F. Vestroni, Plane wave solutions and modal analysis in higher order shear and normal deformable plate theories. Journal of Sound and Vibration, 2002. 257(1): p. 6388 .

14. Carrera, E. and M. Petrolo, Guidelines and recommendations to construct theories for metallic and composite plates. AIAA Journal, 2010. 48(12): p. 2852-2866.

15. Qian, L.F., Batra, R.C. and L.M. Chen, Elastostatic deformations of a thick plate by using a higher-order shear and normal deformable plate theory and two meshless local Petrov-Galerkin (MLPG) methods. Computer Modeling and Engineering and Science, 2003. 4(1): p. 161-175.

16. Qian, L.F., Batra, R.C. and L.M. Chen, Free and Forced Vibrations of Thick Rectangular Plates 
using Higher-Order Shear and Normal Deformable Plate Theory and Meshless Petrov-Galerkin (MLPG) Method. Computer Modeling and Engineering and Science, 2003. 4(5): p. 519-534.

17. Whitney, J.M. and A.W. Leissa, Analysis of heterogeneous anisotropic plates. Journal of Applied Mechanics, 1969. 36(2): p. 261-266.

18. Lo, K.H., Christensen, R.M. and E.M. Wu, A high-order theory of plate deformation-part 2: laminated plates. Journal of Applied Mechanics, 1977. 44(4): p. 669-676.

19. Murty, A.K., Higher order theory for vibrations of thick plates. AIAA Journal, 1977. 15(12): p. 1823-1824.

20. Reddy, J.N., A simple higher-order theory for laminated composite plates. Journal of Applied Mechanics, 1984. 51(4): p. 745-752.

21. Reissner, E., On a mixed variational theorem and on shear deformable plate theory. International Journal for Numerical Methods in Engineering, 1986. 23(2): p. 193-198.

22. Lekhnitskii, S., Strength calculation of composite beams. Vestnik inzhen i tekhnikov, 1935. 9.

23. Ambartsumian, S.A., Theory of anisotropic shells. 1961: State Publishing House for Physical and Mathematical Literature.

24. Rasskazov, A.O., Theory of multilayer orthotropic shallow shells. Soviet Applied Mechanics, 1976. 12(11): p. 1131-1136.

25. Carrera, E., Mixed layer-wise models for multilayered plates analysis. Composite Structures, 1998. 43(1): p. 57-70.

26. Di Sciuva, M., An improved shear-deformation theory for moderately thick multilayered anisotropic shells and plates. Journal of Applied Mechanics, 1987. 54(3): p. 589-596.

27. Carrera, E., Historical review of Zig-Zag theories for multilayered plates and shells. Applied Mechanics Reviews, 2003. 56(3): p. 287-308.

28. Ren, J.G., A new theory of laminated plate. Composites Science and Technology, 1986. 26(3): p. 225-239.

29. Ren, J.G., Bending theory of laminated plate. Composites Science and Technology, 1986. 27(3): p. 225-248.

30. Ren, J.G. and D.R.J. Owen, Vibration and buckling of laminated plates. International Journal of Solids and Structures, 1989. 25(2): p. 95-106.

31. Ambartsumian, S.A., On a theory of bending of anisotropic plates, Investiia Akad Nauk SSSR, Ot Tekh Nauk, No. 4., 1958.

32. Ambartsumian, S.A., On a general theory of anisotropic shells. Journal of Applied Mathematics and Mechanics, 1958. 22(2): p. 305-319.

33. Whitney, J., The effect of transverse shear deformation on the bending of laminated plates. 
Journal of Composite Materials, 1969. 3(3): p. 534-547.

34. Rath, B.K and Y.C. Das, Vibration of layered shells, Journal of Sound and Vibration, 1973. 28: p. 737-757.

35. Ambartsumian, S.A., Contributions to the theory of anisotropic layered shells, Applied Mechanics Reviews, 1962. 15(4): p. 245-249.

36. Ambartsumian, S.A., Nontraditional theories of shells and plates, Applied Mechanics Reviews, 2002. 55(5): p. 35-44.

37. Ghugal, Y. and R. Shimpi, A review of refined shear deformation theories for isotropic and anisotropic laminated beams. Journal of Reinforced Plastics and Composites, 2001. 20(3): p. 255272.

38. Reddy, J.N., An evaluation of equivalent-single-layer and layerwise theories of composite laminates. Composite Structures, 1993. 25(1-4): p. 21-35.

39. Reddy, J.N. and R.A. Arciniega, Shear deformation plate and shell theories: from Stavsky to present. Mechanics of Advanced Materials and Structures, 2004. 11(6): p. 535-582.

40. Rohwer, K., Friedrichs, S. and C. Wehmeyer, Analyzing laminated structures from fibrereinforced composite material - An assessment. Technische Mechanik, 2005. 25(1): p. 59-79.

41. Pagano, N.J., Exact solutions for rectangular bidirectional composites and sandwich plates, Journal of Composite Materials, 1970. 4(1): p. 20-34.

42. Rohwer, K., Application of higher order theories to the bending analysis of layered composite plates. International Journal of Solids and Structures, 1992. 29(1): p. 105-119.

43. Noor, A.K., Burton, W.S. and J.M. Peters, Predictor-corrector procedures for stress and free vibration analyses of multilayered composite plates and shells. Computer Methods in Applied Mechanics and Engineering, 1990. 82: p. 341-363.

44. Noor, A.K., Burton, W.S. and J.M. Peters, Assessment of computational models for multilayered composite cylinders. International Journal of Solids and Structures, 1991. 27(10): p. 1269-1286.

45. Malik, M. and A.K. Noor, Accurate determination of transverse normal stresses in hybrid laminated panels subjected to electro-thermo-mechanical loadings. International Journal for Numerical Methods in Engineering, 2000. 47(1-3): p. 477-495.

46. Chaudhuri, R.A., and P. Seide, An approximate semi-analytical method for prediction of interlaminar shear stresses in an arbitrarily laminated thick plate. Computers and Structures, 1987. 25(4): p. 627-636.

47. Engelstad, S.P., Reddy, J.N. and N.F. Knight, Postbuckling response and failure prediction of graphite-epoxy plates loaded in compression. AIAA Journal, 1992. 30(8): p. 2106-2113.

48. Byun, C. and Kapania, R.K. Prediction of interlaminar stresses in laminated plates using global orthogonal interpolation polynomials. AIAA Journal, 1992. 30(11): pp. 2740-2749. 
49. Hartman, T.B., Hyer, M.W. and M.W. Case, Geometrically nonlinear stress recovery in composite laminates. AIAA Journal, 2012. 50(5): p. 1156-1168.

50. Park, B.C., Park, J.W. and Y.H. Kim, Stress recovery in laminated composite and sandwich panels undergoing finite rotation. Composite Structures, 2003. 59(2): p. 227-235.

51. Türkmen, H.S. and Z. Mecitoğlu, Dynamic response of a stiffened laminated composite plate subjected to blast load. Journal of Sound and Vibration, 1999. 221(3): p. 371-389.

52. Barlow, J., Optimal stress locations in finite element models, International Journal for Numerical Methods in Engineering, 1976. 10(2): p. 243-251.

53. Vel, S.S. and R.C. Batra, Analytical solution for rectangular thick laminated plates subjected to arbitrary boundary conditions. AIAA journal, 1999. 37(11): p. 1464-1473.

54. Batra, R.C. and J. Xiao, Finite deformations of curved laminated St. Venant-Kirchhoff beam using layer-wise third order shear and normal deformable beam theory (TSNDT). Composite Structures, 2013. 97: p. 147-164.

\section{Appendix A}

The matrices $\mathbf{Z}_{\mathbf{i}}$ (i $\left.=0,1,2,3\right)$ and the operator matrix $\mathbf{L}$ appearing in Eq. (3) are given by Eqs. (A.1) and (A.2), respectively.

$$
\mathbf{Z}_{\mathrm{i}}=\left[\begin{array}{ccccccccc}
\mathrm{X}_{3}^{\mathrm{i}} & 0 & 0 & 0 & 0 & 0 & 0 & 0 & 0 \\
0 & \mathrm{X}_{3}^{\mathrm{i}} & 0 & 0 & 0 & 0 & 0 & 0 & 0 \\
0 & 0 & 0 & 0 & \mathrm{i} \mathrm{X} \mathrm{X}_{3}^{\mathrm{i}-1} & 0 & 0 & 0 & 0 \\
0 & 0 & 0 & 0 & 0 & \mathrm{i} \mathrm{X} \mathrm{X}_{3}^{\mathrm{i}-1} & 0 & \mathrm{X}_{3}^{\mathrm{i}} & 0 \\
0 & 0 & 0 & 0 & 0 & 0 & \mathrm{ix} \mathrm{X}_{3}^{\mathrm{i}-1} & 0 & \mathrm{X}_{3}^{\mathrm{i}} \\
0 & 0 & \mathrm{X}_{3}^{\mathrm{i}} & \mathrm{X}_{3}^{\mathrm{i}} & 0 & 0 & 0 & 0 & 0
\end{array}\right]
$$




$$
\mathbf{L}=\left[\begin{array}{ccc}
\frac{\partial}{\partial \mathrm{X}_{1}} & 0 & 0 \\
0 & \frac{\partial}{\partial \mathrm{X}_{2}} & 0 \\
\frac{\partial}{\partial \mathrm{X}_{2}} & 0 & 0 \\
0 & \frac{\partial}{\partial \mathrm{X}_{1}} & 0 \\
0 & 0 & 1 \\
0 & 1 & 0 \\
1 & 0 & 0 \\
0 & 0 & \frac{\partial}{\partial \mathrm{X}_{2}} \\
0 & 0 & \frac{\partial}{\partial \mathrm{X}_{1}}
\end{array}\right]
$$

\section{Appendix B}

The degrees of freedom associated with the $i^{\text {th }}$ node are indicated by adding a superscript $i$ to variables in Eq. (3.2). That is,

$$
\mathbf{d}_{\mathbf{0}}^{\mathrm{i}}=\left[\begin{array}{lll}
\mathrm{u}_{10}^{\mathrm{i}} & \mathrm{u}_{20}^{\mathrm{i}} & \mathrm{u}_{20}^{\mathrm{i}}
\end{array}\right]^{\mathrm{T}}, \mathbf{d}_{\mathbf{1}}^{\mathrm{i}}=\left[\begin{array}{lll}
\mathrm{u}_{11}^{\mathrm{i}} & \mathrm{u}_{21}^{\mathrm{i}} & \mathrm{u}_{31}^{\mathrm{i}}
\end{array}\right]^{\mathrm{T}}, \mathbf{d}_{\mathbf{2}}^{\mathrm{i}}=\left[\begin{array}{lll}
\mathrm{u}_{12}^{\mathrm{i}} & \mathrm{u}_{22}^{\mathrm{i}} & \mathrm{u}_{32}^{\mathrm{i}}
\end{array}\right]^{\mathrm{T}} \text { and } \mathbf{d}_{\mathbf{3}}^{\mathrm{i}}=\left[\begin{array}{lll}
\mathrm{u}_{13}^{\mathrm{i}} & \mathrm{u}_{23}^{\mathrm{i}} & \mathrm{u}_{33}^{\mathrm{i}}
\end{array}\right]^{\mathrm{T}}
$$

Thus the vector of generalized variables $\mathbf{d}_{\mathbf{j}}(\mathbf{j}=0,1,2,3)$ of a point in an element e can be expressed in terms of the 24-D vector $\mathbf{d}_{\mathbf{j}}^{\mathbf{e}}=\left[\mathbf{d}_{\mathbf{j}}^{\mathbf{1}}, \mathbf{d}_{\mathbf{j}}^{\mathbf{2}}, \ldots, \mathbf{d}_{\mathbf{j}}^{\mathbf{8}}\right]$ containing values of $\mathbf{d}_{\mathbf{j}}$ at the 8nodes of the element as follows:

$$
\mathbf{d}_{\mathbf{j}}=\boldsymbol{\Phi} \mathbf{d}_{\mathbf{j}}^{\mathbf{e}}(\mathbf{j}=0,1,2,3)
$$

where $\Phi$ is a (3 x 24) matrix containing shape functions $\left(\psi_{1}, \psi_{2}, \ldots, \psi_{8}\right)$ associated with the 8 nodes of the element given by

$\boldsymbol{\Phi}=\left[\begin{array}{lll}\psi_{1} \mathbf{I} & \psi_{2} \mathbf{I} \ldots & \psi_{8} \mathbf{I}\end{array}\right]$

in which $\mathbf{I}$ is a $(3 \times 3)$ identity matrix. 
Substituting for $\mathbf{d}_{\mathbf{m}}(\mathrm{m}=0,1,2,3)$ from Eq. (B.2) into Eq. (10), the first variation of the total potential energy of a typical element is given by

$$
\delta \Pi^{\mathrm{e}}=\delta \overline{\mathbf{d}}_{\mathbf{i}}^{\mathbf{e}^{\mathrm{T}}} \mathbf{K}_{\mathrm{ij}}^{\mathbf{e}} \overline{\mathbf{d}}_{\mathbf{j}}^{\mathbf{e}}-\delta \overline{\mathbf{d}}_{\mathrm{i}}^{\mathrm{e}}\left(\mathbf{T}_{\mathbf{i}}^{+\mathbf{e}}+\mathbf{T}_{\mathbf{i}}^{-\mathbf{e}}+\mathbf{P}_{\mathbf{i}}^{+\mathbf{e}}+\mathbf{P}_{\mathbf{i}}^{-\mathbf{e}}+\mathbf{Q}_{\mathbf{i}}^{+\mathbf{e}}+\mathbf{Q}_{\mathbf{i}}^{-\mathbf{e}}\right)=0 \quad(\mathrm{i}, \mathrm{j}=0,1,2,3)
$$

The elemental stiffness matrices $\mathbf{K}_{\mathrm{ij}}^{\mathrm{e}}$ and the elemental load vectors $\mathbf{T}_{\mathbf{i}}^{ \pm \mathbf{e}}, \mathbf{P}_{\mathbf{i}}^{ \pm \mathbf{e}}, \mathbf{Q}_{\mathbf{i}}^{ \pm \mathbf{e}}$ $(\mathrm{i}, \mathrm{j}=0,1,2,3)$ appearing in Eq. (B.3) are given by

$$
\begin{aligned}
& \mathbf{K}_{\mathbf{i j}}^{\mathbf{e}}=\int_{\mathrm{X}_{2}^{\mathrm{e}}}^{\mathrm{X}_{2}^{\mathrm{e}+1}} \int_{\mathrm{X}_{1}^{\mathrm{e}}}^{\mathrm{X}_{1}^{\mathrm{e}+1}} \mathbf{B}^{\mathrm{T}} \mathbf{D}_{\mathbf{i j}} \mathbf{B} \mathrm{d} \mathrm{X}_{1} \mathrm{~d} \mathbf{X}_{2} \text {, where } \mathbf{B}=\mathbf{L} \boldsymbol{\Phi} \\
& \mathbf{T}_{\mathbf{i}}^{ \pm \mathbf{e}}=\int_{\mathrm{X}_{2}^{\mathrm{e}}}^{\mathrm{X}_{2}^{\mathrm{e}+1}} \int_{\mathrm{X}_{1}^{\mathrm{e}}}^{\mathrm{X}_{1}^{\mathrm{e}+1}} \mathrm{X}_{3}^{\mathrm{i}} \mathbf{N}^{\mathrm{T}} \mathbf{f}^{ \pm} \mathrm{d} \mathrm{X}_{1} \mathrm{~d} \mathrm{X}_{2}, \mathbf{P}_{\mathbf{i}}^{ \pm \mathbf{e}}=\int_{\mathrm{X}_{2}^{\mathrm{e}}}^{\mathrm{X}_{2}^{\mathrm{e}+1}} \int_{-\mathrm{h} / 2}^{\mathrm{h} / 2} \mathrm{X}_{3}^{\mathrm{i}} \mathbf{N}^{\mathrm{T}} \mathbf{p}^{ \pm} \mathrm{d} \mathrm{X}_{3} \mathrm{~d} \mathrm{X}_{2}, \mathbf{Q}_{\mathbf{i}}^{ \pm \mathbf{e}}=\int_{\mathrm{X}_{1}^{\mathrm{e}}}^{\mathrm{X}_{1}^{\mathrm{e}+1} / 2} \int_{-\mathrm{h} / 2}^{\mathrm{h} / 2} \mathrm{X}_{3}^{\mathrm{i}} \mathbf{N}^{\mathrm{T}} \mathbf{q}^{ \pm} \mathrm{d} \mathrm{X}_{3} \mathrm{~d} \mathrm{X}_{1}
\end{aligned}
$$

where $\mathbf{f}^{+}$and $\mathbf{f}^{-}$are the surface tractions prescribed on the top and the bottom surfaces, respectively; and $\mathbf{p}^{+}, \mathbf{p}^{-}, \mathbf{q}^{+}$and $\mathbf{q}^{-}$are the surface tractions prescribed on the edge surfaces, $\mathrm{X}_{1}=a, \mathrm{X}_{1}=0, \mathrm{X}_{2}=b$ and $\mathrm{X}_{2}=0$, respectively.

Recalling that variations in generalized displacements are arbitrary except at nodes where they are prescribed, we get the following equilibrium equations for an FE:

$$
\begin{aligned}
& \mathbf{K}_{\mathbf{0 0}}^{\mathrm{e}} \overline{\mathbf{d}}_{\mathbf{0}}^{\mathrm{e}}+\frac{1}{2}\left(\mathbf{K}_{\mathbf{0 1}}^{\mathrm{e}}+\mathbf{K}_{\mathbf{1 0}}^{\mathrm{e}}{ }^{\mathrm{T}}\right) \overline{\mathbf{d}}_{\mathbf{1}}^{\mathrm{e}}+\frac{1}{2}\left(\mathbf{K}_{\mathbf{0 2}}^{\mathrm{e}}+\mathbf{K}_{\mathbf{2 0}}^{\mathrm{e} \mathrm{T}}\right) \overline{\mathbf{d}}_{\mathbf{2}}^{\mathrm{e}}+\frac{1}{2}\left(\mathbf{K}_{\mathbf{0 3}}^{\mathrm{e}}+\mathbf{K}_{\mathbf{3 0}}^{\mathrm{e}}{ }^{\mathrm{T}}\right) \overline{\mathbf{d}}_{\mathbf{3}}^{\mathrm{e}}=\mathbf{F}_{\mathbf{0}}^{\mathrm{e}} \\
& \frac{1}{2}\left(\mathbf{K}_{10}^{\mathbf{e}}+\mathbf{K}_{\mathbf{0 1}}^{\mathbf{e}{ }^{\mathrm{T}}}\right) \overline{\mathbf{d}}_{\mathbf{0}}^{\mathbf{e}}+\mathbf{K}_{11}^{\mathbf{e}} \overline{\mathbf{d}}_{\mathbf{1}}^{\mathbf{e}}+\frac{1}{2}\left(\mathbf{K}_{12}^{\mathbf{e}}+\mathbf{K}_{21}^{\mathbf{e}^{\mathrm{T}}}\right) \overline{\mathbf{d}}_{\mathbf{2}}^{\mathbf{e}}+\frac{1}{2}\left(\mathbf{K}_{13}^{\mathbf{e}}+\mathbf{K}_{31}^{\mathbf{e} T}\right) \overline{\mathbf{d}}_{3}^{\mathbf{e}}=\mathbf{F}_{1}^{\mathbf{e}}
\end{aligned}
$$

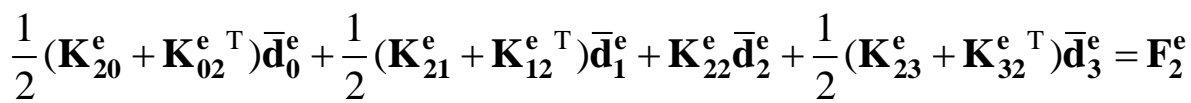

$$
\begin{aligned}
& \frac{1}{2}\left(\mathbf{K}_{\mathbf{3 0}}^{\mathbf{e}}+\mathbf{K}_{\mathbf{0 3}}^{\mathbf{e}^{\mathrm{T}}}\right) \overline{\mathbf{d}}_{\mathbf{0}}^{\mathbf{e}}+\frac{1}{2}\left(\mathbf{K}_{\mathbf{3 1}}^{\mathbf{e}}+\mathbf{K}_{\mathbf{1 3}}^{\mathbf{e}^{\mathrm{T}}}\right) \overline{\mathbf{d}}_{\mathbf{1}}^{\mathrm{e}}+\frac{1}{2}\left(\mathbf{K}_{\mathbf{3 2}}^{\mathbf{e}}+\mathbf{K}_{\mathbf{2 3}}^{\mathbf{e}}\right) \overline{\mathbf{d}}_{\mathbf{2}}^{\mathbf{e}}+\mathbf{K}_{\mathbf{3 3}}^{\mathbf{e}} \overline{\mathbf{d}}_{\mathbf{3}}^{\mathbf{e}}=\mathbf{F}_{\mathbf{3}}^{\mathbf{e}}
\end{aligned}
$$

where

$$
\mathbf{F}_{\mathbf{i}}^{\mathbf{e}}=\mathbf{T}_{\mathbf{i}}^{+\mathbf{e}}+\mathbf{T}_{\mathbf{i}}^{-\mathbf{e}}+\mathbf{P}_{\mathbf{i}}^{+\mathbf{e}}+\mathbf{P}_{\mathbf{i}}^{-\mathbf{e}}+\mathbf{Q}_{\mathbf{i}}^{+\mathbf{e}}+\mathbf{Q}_{\mathbf{i}}^{-\mathbf{e}}(\mathbf{i}=0,1,2,3)
$$

Equations (B.6) are assembled using the standard technique to obtain Eq. (11) given in section 2 . 
The expressions for $\mathbf{K}, \mathbf{U}$, and $\mathbf{F}$ in Eq. (11) are given by

$$
\begin{aligned}
\mathbf{K} & =\left[\begin{array}{cccc}
\mathbf{K}_{\mathbf{0 0}} & \frac{1}{2}\left(\mathbf{K}_{\mathbf{0 1}}+\mathbf{K}_{\mathbf{1 0}}^{\mathrm{T}}\right) & \frac{1}{2}\left(\mathbf{K}_{\mathbf{0 2}}+\mathbf{K}_{\mathbf{2 0}}^{\mathrm{T}}\right) & \frac{1}{2}\left(\mathbf{K}_{\mathbf{0 3}}+\mathbf{K}_{\mathbf{3 0}}^{\mathrm{T}}\right) \\
\frac{1}{2}\left(\mathbf{K}_{\mathbf{1 0}}+\mathbf{K}_{\mathbf{0 1}}^{\mathrm{T}}\right) & \mathbf{K}_{\mathbf{1 1}} & \frac{1}{2}\left(\mathbf{K}_{\mathbf{1 2}}+\mathbf{K}_{\mathbf{2 1}}^{\mathrm{T}}\right) & \frac{1}{2}\left(\mathbf{K}_{\mathbf{1 3}}+\mathbf{K}_{\mathbf{3 1}}^{\mathrm{T}}\right) \\
\frac{1}{2}\left(\mathbf{K}_{\mathbf{2 0}}+\mathbf{K}_{\mathbf{0 2}}^{\mathrm{T}}\right) & \frac{1}{2}\left(\mathbf{K}_{\mathbf{2 1}}+\mathbf{K}_{\mathbf{1 2}}^{\mathrm{T}}\right) & \mathbf{K}_{\mathbf{2 2}} & \frac{1}{2}\left(\mathbf{K}_{\mathbf{2 3}}+\mathbf{K}_{\mathbf{3 2}}^{\mathrm{T}}\right) \\
\frac{1}{2}\left(\mathbf{K}_{\mathbf{3 0}}+\mathbf{K}_{\mathbf{0 3}}^{\mathrm{T}}\right) & \frac{1}{2}\left(\mathbf{K}_{\mathbf{3 1}}+\mathbf{K}_{\mathbf{1 3}}^{\mathrm{T}}\right) & \frac{1}{2}\left(\mathbf{K}_{\mathbf{3 2}}+\mathbf{K}_{\mathbf{2 3}}^{\mathrm{T}}\right) & \mathbf{K}_{\mathbf{3 3}}
\end{array}\right] \\
\mathbf{U} & =\left[\begin{array}{l}
\mathbf{U}_{\mathbf{0}} \\
\mathbf{U}_{\mathbf{1}} \\
\mathbf{U}_{\mathbf{2}} \\
\mathbf{U}_{\mathbf{3}}
\end{array}\right], \quad \mathbf{F}=\left[\begin{array}{l}
\mathbf{F}_{\mathbf{0}} \\
\mathbf{F}_{\mathbf{1}} \\
\mathbf{F}_{\mathbf{2}} \\
\mathbf{F}_{\mathbf{3}}
\end{array}\right]
\end{aligned}
$$

in which $\mathbf{U}_{\mathbf{0}}, \mathbf{U}_{\mathbf{1}}, \mathbf{U}_{\mathbf{2}}$ and $\mathbf{U}_{\mathbf{3}}$ are the global vectors of generalized displacements, slopes, curvature, and curvature gradients, respectively. 